\title{
Review \\ Unveiling the Bioactive Potential of Fresh Fruit and Vegetable Waste in Human Health from a Consumer Perspective
}

\author{
Jorge A. M. Pereira ${ }^{1, *(\mathbb{D})}$, Cristina V. Berenguer ${ }^{1}\left(\mathbb{D}\right.$, Carolina F. P. Andrade $^{1}$ and José S. Câmara ${ }^{1,2} \mathbb{D}$ \\ 1 CQM-Centro de Química da Madeira, Campus da Penteada, Universidade da Madeira, 9020-105 Funchal, \\ Portugal; cristina.berenguer@staff.uma.pt (C.V.B.); carolinafatimaandrade@hotmail.com (C.F.P.A.); \\ jsc@staff.uma.pt (J.S.C.) \\ 2 Departamento de Química, Faculdade de Ciências Exatas e da Engenharia, Campus da Penteada, \\ Universidade da Madeira, 9020-105 Funchal, Portugal \\ * Correspondence: jorge.pereira@staff.uma.pt; Tel.: +351-291705119; Fax: +351-291705149
}

check for updates

Citation: Pereira, J.A.M.; Berenguer, C.V.; Andrade, C.F.P.; Câmara, J.S. Unveiling the Bioactive Potential of Fresh Fruit and Vegetable Waste in Human Health from a Consumer Perspective. Appl. Sci. 2022, 12, 2747. https://doi.org/10.3390/app12052747

Academic Editor: Luca Mazzoni

Received: 11 February 2022

Accepted: 2 March 2022

Published: 7 March 2022

Publisher's Note: MDPI stays neutral with regard to jurisdictional claims in published maps and institutional affiliations.

Copyright: (c) 2022 by the authors. Licensee MDPI, Basel, Switzerland. This article is an open access article distributed under the terms and conditions of the Creative Commons Attribution (CC BY) license (https:// creativecommons.org/licenses/by/ $4.0 /)$.

\begin{abstract}
Food supply disruption and shortage verified during the current pandemic events are a scenario that many anticipate for the near future. The impact of climate changes on food production, the continuous decrease in arable land, and the exponential growth of the human population are important drivers for this problem. In this context, adding value to food waste is an obvious strategy to mitigate food shortages, but there is a long way to go in this field. Globally, it is estimated that one-third of all food produced is lost. This is certainly due to many different factors, but the lack of awareness of the consumer about the nutritional value of certain foods parts, namely peels and seeds, is certainly among them. In this review, we will unveil the nutritional and bioactive value of the waste discarded from the most important fresh fruit and vegetables consumed worldwide as a strategy to decrease food waste. This will span the characterization of the bioactive composition of selected waste from fruits and vegetables, particularly their seeds and peels, and their possible uses, whether in our diet or recycled to other ends.
\end{abstract}

Keywords: bioactive compounds; fruit waste; vegetable waste; health; disease; peels; seeds

\section{Introduction}

According to UN estimates, the world's population will reach 9.8 billion by 2050, nearly $20 \%$ higher than today [1]. This exponential increase combined with continuous climate changes, water scarcity, and decreasing of agricultural areas constitutes societal and global problems that challenge food production for the next generations. With current global trends in diets, the exponentially growing population and considering the several millions of tons of food that are lost and wasted every year in different steps of the food chain, including production, post-harvesting, processing, and distribution, in 2050 50-60\% more food will be needed than today to feed everyone. These overwhelming numbers clearly show that food challenges and planet sustainability are intrinsically bound and must determine urgent measures to mitigate them. It will be important to develop strategies to produce more food and better food with less waste. In addition, the implementation of sustainable food production systems through the optimization of food processes will be essential to achieve a better environmental footprint, lower production costs, and improve the quality and nutritional value of food (Figure 1).

Given these facts, the European Commission established food waste as one of the priority areas of the Action Plan for the European Circular Economy Strategy [2]. This includes a zero-waste strategy envisaging agri-food waste to reduce environmental pollution. This strategy is based on the extraction of compounds from food waste that have a high demand or innovative applications with potentially high economic return in different industrial sectors, such as nutraceutical, cosmetic, and pharmaceutical industries [3,4]. In this context, the extraction of phytochemicals from fruit and vegetable waste able to substitute the 
use of synthetic preservatives in food products and confer additional health protection effects to our diet is of utmost importance [3-6]. However, these strategies mostly target the food industries and food chain stakeholders and not the final consumer. Remarkably, almost half of the losses and waste in fruits and vegetables are caused by human eating habits, being the second cause of greenhouse gas emission. Statistically, this is equivalent to 1.3 billion tons of wasted foods causing the emission of around 4.4 gigatons of greenhouse gas [1]. This means that every one of us, as final consumers, has important responsibilities to share when a great part of food waste occurs on the supermarket shelves and in our kitchens and fridges. This includes the fruits and vegetables that are rejected because of their non-standardized measures or defects, commonly known as ugly foods, but also the peels, seeds, rinds, or cores, which despite being edible and rich in nutrients and bioactive compounds, are discarded to the food waste bin. In this context, this review targets the traditional discarded parts of the most relevant fruits and vegetables traded worldwide, aiming to show their nutritional and bioactive composition as a strategy to potentiate their dietary use by the consumers, which will be shown with illustrative examples.

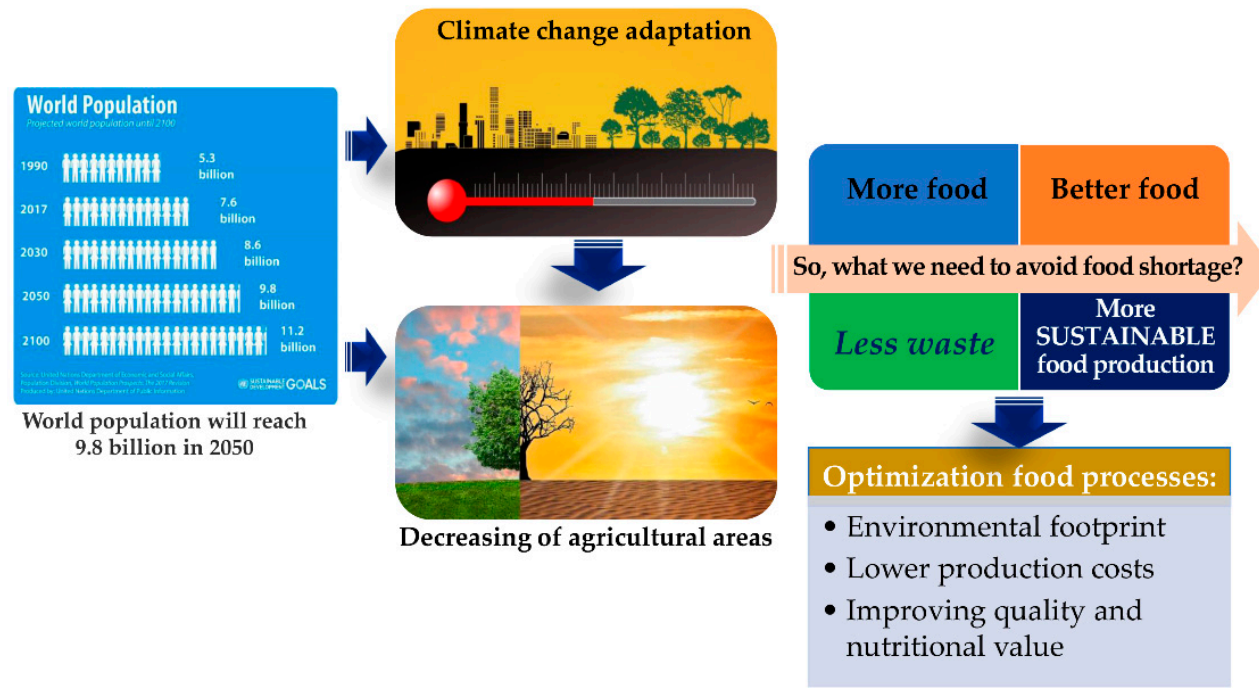

Figure 1. Global and societal challenges in the production of foods for the next generations.

\section{Global Trade of Fresh Fruits and Vegetables}

Ensuring food chain supplies worldwide is a huge task that influences the world food trade market with a focus on essential foods for human nutrition including fruits, vegetables, and processed plant-based foods such as juices and jams. The challenges of meeting future food needs will require a concerted effort from stakeholders, food sector enterprises, governments, and governmental institutions, to improve the functioning of food and agricultural markets. After the peak reached in 2018, global volumes of fruit and vegetable trade have followed a downward trend down to 87 million tons of global trade in 2020. The observed tendency can be explained as an overall deceleration in the growth of booming categories, accentuated by the COVID-19 pandemic's disruptions which caused logistical difficulties worldwide in several ways. This situation had a significant impact on the marketing of vegetables and fresh fruits due to a sharp decrease in out-of-home consumption related to the closure of catering outlets and restaurants, and the limited availability of workers. In turn, European Union (EU) exports outside of the Single Market went down to record low levels to 3.9 million tons, although value continued increasing up to EUR 4.6 billion. Europe constitutes an attractive and diverse target market for fresh fruits and vegetables for suppliers in developing countries. The large volumes necessary for the 500 million consumers are responsible for $44 \%$ of the global trade value, representing over EUR 60 billion. Moreover, the overall demand is relatively stable, while prices and shortterm needs change regularly. The international trade of fruits and vegetables, however, continues to be dominated by the United States (US). The major destinations and sources 
of supply in fruits and vegetables remain in the EU, the North American Free Trade Agreement (NAFTA) area, and Asia (East, Southeast, and South). A significant share of their trade is still dominated by intraregional trade, particularly that of the EU. However, these regions depend on Southern Hemisphere countries-Chile, South Africa, and New Zealand-for imports of off-season fresh fruits and juices, and on Equatorial regions for bananas. Germany (9\%), the Netherlands (6\%) the United Kingdom (6\%), and France (5\%) are the major importer markets, while Spain and the Netherlands are the largest exporters. In Asia, China is the largest exporter and Japan is the largest importer while within the NAFTA countries Mexico is the main exporter and the US accounts for most of the fruit and vegetable imports [7]. In Europe, Spain and Italy gather $40 \%$ of vegetable production and more than $50 \%$ of fruit (including citrus). Italy remains the largest European fruitand vegetable-producing country. Asia is the leading producer of vegetables with a $61 \%$ total volume output and a yearly growth of $51 \%$. However, the US continues to lead in the export of fresh fruits and vegetables worldwide with oranges, grapes, and tomatoes. Brazil dominates the international trade of frozen orange juice concentrate, while Chile has become the major fresh fruit exporter with a production volume of $45 \%$. World fruit production went up 54\% between 2019 and 2000, to 883 million tonnes, which represents an increase of 311 million tonnes. Bananas and plantains (18\%), watermelons $(11 \%)$, apples $(10 \%)$, oranges $(9 \%)$, and grapes $(9 \%)$, accounted for $57 \%$ of the total fruit production in 2019 (Figure 2a). Despite the significant increase in fruit production, $6 \%$ between 2000 and 2019, which corresponds to an increase of more than 300 million tons, the production of the main fruit varieties during the same period remained almost constant (Figure 2a).

(a) Fruits

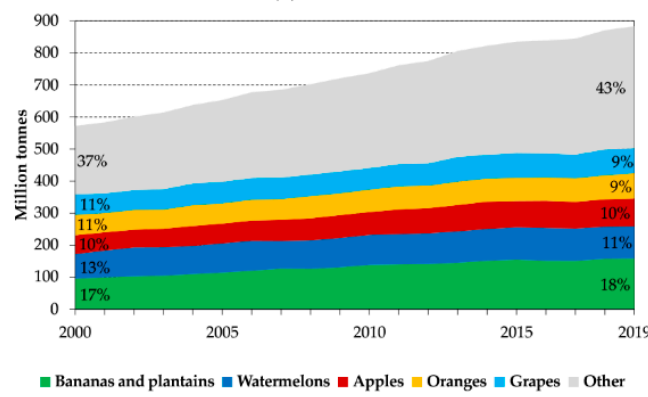

(b) Vegetables

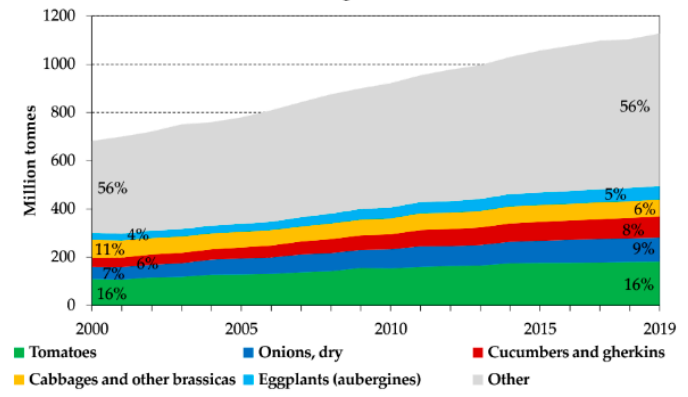

Figure 2. World production of fruits (a) and vegetables (b), main commodities (adapted from [7]).

Compared to fruit production, the world vegetable production grew faster between 2000 and 2019 to 1128 million tonnes in 2019 , corresponding to an increase of $65 \%$. The five main vegetable species, tomatoes $(16 \%$ in 2019), onions (9\%), cucumbers and gherkins $(8 \%)$, cabbages $(6 \%)$, and eggplants $(5 \%)$, accounted for $44 \%$ of the total production in 2019 (Figure $2 b$ ). The share of cucumbers and gherkins, onions, and eggplants increased, while that of tomatoes remained stable and that of cabbages almost halved (Figure 2b).

\section{Bioactive Compounds in Fresh Fruits and Vegetables}

\subsection{Protection and Disease Prevention}

The human diet has a significant role in the prevention of diseases, particularly those that are highly modulated by behavioral factors and lifestyle choices, such as cardiovascular (CVDs), neurodegenerative (NDs), and oncologic diseases (ODs), gastrointestinal disorders, or diabetes $[3,8]$. Such protection is certainly related to fruit and vegetable consumption because they are highly represented in the human diet, and several portions of fruits and vegetables per day are recommended to keep a healthy lifestyle and dietary habits regardless of the different dietary regimes adopted [9]. This importance is supported by the epidemiological evidence of the inverse correlation between the regular consumption of fruits and vegetables and the risk for developing one or more of the most prevalent diseases of the modern day, namely CVDs, NDs, and ODs [10]. Effectively, numerous studies link 
the preventive potential of nutrition to the bioactive compounds derived from different food products [11]. Accordingly, dietary interventions involving a shift to well-balanced diets, rich in bioactive compounds, have been demonstrated to reduce the prevalence of several health conditions, including type 2 diabetes mellitus, CVDs, obesity, or metabolic syndromes, as well as neuroprotection and reduced risk of central nervous system pathologies, such as Alzheimer's disease (AD) and dementia [12-14]. This indicates that bioactive compounds could influence the underlying pathological mechanisms of neurodegenerative diseases, and improve lipid profile, glucose metabolism, and blood pressure, due to their antioxidation, antidiabetic, antiplatelet, and antiaggregating properties [11,13-17]. Broadly, these bioactive compounds are naturally occurring essential and non-essential compounds that have been shown to affect human health $[3,4,18,19]$, while meeting the basic nutritional requirements [20]. This includes diverse classes of compounds, such as probiotics, dietary fibers, fat-soluble vitamins (vitamins A, D, E, and K), essential omega-3 fatty acids, phytoestrogens, and several other phytochemicals with diverse structures and bioactivities, namely carotenoids (lutein and zeaxanthin), flavonoids (hesperetin, quercetin, genistein, and kaempferol), and phenolic acids (caffeic, gallic, ferulic, and chlorogenic acid) $[4,17,21]$.

\subsubsection{Polyphenols}

Polyphenols are the most relevant secondary metabolites in fruits and vegetables. Their structure is characterized by at least one aromatic ring with one or more hydroxyl groups attached, ranging from simple to highly polymerized molecules [22]. These compounds show potential benefits for cardiovascular and metabolic health, cancer prevention, and antioxidant, anti-inflammatory, and antimicrobial activities [23]. Examples of phenolic compounds include oleuropein and hydroxytyrosol, both strong antioxidants, that help protect nerve cells from neurotoxin-induced apoptosis [24] and can act as scavengers of reactive oxygen species (ROS) $[17,25]$, respectively. Curcumin is a phenolic pigment that has been shown to possess neuroprotective properties, due to its potent antioxidant activity. This compound also reduces protein oxidation products and attenuates inflammation by inhibition of lipoxygenase enzymes and microglial activity [26]. Polyphenols can be further considered as flavonoids that include several subclasses such as flavonols and flavanols, flavones and isoflavones, flavanones, and anthocyanidins. The non-flavonoid polyphenols are mainly represented by phenolic acids, stilbenes, lignans, and tannins (reviewed in [27]).

\subsubsection{Flavonoids}

Flavonoids are the most common polyphenolic compounds in plant food and act as free radical and ROS scavengers [28]. They are also known to exhibit antibacterial, antiviral, anti-inflammatory, antiallergenic, antithrombotic, and vasodilatory activities (reviewed in [27]). The benefits from their consumption are related to their antioxidant and chelating properties, as they exhibit antimutagenic and antitumoral activities, preventing the spread of bacterial or tumor metastases [8,29]. Flavon-3-ol and epigallocatechin-3-gallate are examples of flavonoids that are cardioprotective antioxidants [14]. Anthocyanins are a type of flavonoid responsible for the red, violet, and blue color of many fruits and vegetables. These compounds act as photoprotectors preventing the damage caused by free radicals during photosynthesis. Anthocyanins possess anti-inflammatory activity, inhibiting the expression of inflammatory cytokines [22] and are neuroprotective, as they ameliorate oxidative stress by lowering free radical production and lipid peroxidation [30]. For instance, higher consumption of fruits rich in anthocyanins was linked to a delayed loss of cognitive function $[17,31]$. Quercetin is one of the most abundant flavonols in plants and has been found to have an active role in several cancers and CVDs and NDDs. Myricetin is another flavonol abundant in fruits, well known for its effective action against diabetes mellitus [32,33]. Genistein, an isoflavone, has a preventative effect on AD, as it reduces oxidative stress by ROS inactivation [17,34]. Along with daidzein, these compounds are also used for regulating skin aging problems [22]. 


\subsubsection{Phenolic Acids}

Phenolic acids are the major source of antioxidants in plant foods. They are characterized by a carboxyl group linked to the benzene ring and are divided into hydroxybenzoic acid derivatives and hydroxycinnamic acid derivatives [35]. These compounds are also involved in the reduction of oxidative stress-induced tissue damage due to chronic diseases and anticancer activities [33]. Caffeic, gallic, and chlorogenic acids are examples of phenolic acids, which have antioxidant and antihistamine effects [14,36].

\subsubsection{Stilbenes}

Stilbenes have gained a lot of importance in recent years, due to their antioxidant, antibacterial, antifungal, cardio- and neuroprotective, antiaging, and anticancer properties [33]. For instance, resveratrol exhibits antioxidant, anticancer, anti-inflammatory, and cardioprotective effects [22]. Its antioxidant activity helps maintain cellular health by slowing the aging process [37]. Regarding its anti-inflammatory properties, resveratrol protects the cardiovascular system by reducing the risk of atherosclerosis, platelet aggregation, and myocardial fibrosis. This compound also lowers blood pressure because of its antioxidant properties, through the production of nitric oxide, which causes arterial vasodilation, decreasing blood pressure [14].

\subsubsection{Carotenoids}

Carotenoids, along with phenolic components, are known for their activity against different diseases, including cancer and inflammatory or CVDs. These components have antioxidant and antiradical properties, that can delay, hinder, or inhibit DNA, protein, and lipid oxidation [4]. Carotenoids are present in many fruits and vegetables and are responsible for their yellow, orange, and red colors [17]. Consumption of fruits rich in these compounds is reported to boost immunity and reduce the risk of various diseases, such as cancer, type 2 diabetes, and cardiovascular problems [38], through their antioxidant capacity for ROS [8]. Lycopene and lutein are two of the most prevalent carotenoids in the human diet and both have strong antioxidant capacity. Lycopene is known to protect against prostate cancer, while lutein acts as an inhibitor of age-related muscular degeneration [8,39]. Lakey-Beitia et al. [40], for instance, reported the antiamyloidogenic potential of the carotenoids cryptocapsin, cryptocapsin-5,6-epoxide, and zeaxanthin, and their role in the prevention and treatment of AD [33].

\subsubsection{Glucosinolates}

Glucosinolates are a large group of sulfur-containing compounds present in Brassica vegetables (broccoli and cauliflower). These compounds are degraded into isothiocyanates by the enzymatic action of plant-specific myrosinase or intestinal flora in the body. Isothiocyanates have gained a lot of importance due to their anticarcinogenic properties, especially against colon, rectum, and thyroid cancers [41]. They also act as antioxidants, due to the sulfur atom in their molecule, and have been found to suppress the activity of acetylcholinesterase which is very important in neurotransmission in neurodegenerative diseases $[17,42]$.

\subsubsection{Phytosterols}

Phytosterols have attracted great interest recently. These are naturally occurring plant sterols, present in the non-saponifiable fraction of plant oils. The most common phytosterols in the diet are sitosterol, stigmasterol, and campesterol [20]. These are structurally like cholesterol and compete with it for absorption in the intestinal tract, lowering blood cholesterol levels $[8,43]$. Growing evidence shows that the dietary intake of phytosterols may contribute to cardiovascular protection [41]. 


\subsubsection{Vitamins}

Vitamins are involved in the metabolism of fatty acids, proteins, and carbohydrates. They also participate in the synthesis of various compounds necessary for the proper function of the body. Fat-soluble vitamins, such as vitamins A, D, E, and K, have antioxidant properties and can help reduce the risk of CVDs or neurological diseases, neuropsychiatric disorders, and even cancer [44]. Vitamin A plays an important role in vision, immune function, and regulation of cell growth [45]. Vitamin D improves the intestinal absorption and body retention of calcium, influencing the growth of bone and connective tissues, and has been reported to protect against prostate cancer $[35,46,47]$. Vitamin $\mathrm{E}$ is an antioxidant and has a potent action in brain tissue. This vitamin lowers lipid susceptibility to oxidation, protecting cellular membranes and DNA from free radicals [48]. Vitamin K activates proteins needed for blood coagulation, prevents calcification of arteries and soft tissues, and supports mineralization of bone $[35,49]$.

Fruits and vegetables, including their wasted parts, also contain considerable amounts of several water-soluble vitamins such as thiamine, riboflavin, niacin, and vitamin C [50,51]. Vitamin $C$ has a strong antioxidant activity, protecting our cells from oxidative damage, having a role in cancer prevention and progression that is still under debate [52]. In turn, vitamins from the B complex (thiamine-vitamin B1; riboflavin-vitamin B2; niacinvitamin B3) are important cofactors in biochemical reactions, being essential for normal body growth and development, healthy skin, proper function of nerves and the heart, and red blood cell formation. Vitamins Bs are directly involved in energy metabolism and there is a recent interest in their role in the prevention of heart failure since there is a deficiency in vitamin B in patients affected by this clinical condition [53].

\subsubsection{Polysaccharides and Dietary Fibers}

An important part of the food we ingest does not have any nutritional function, being resistant to enzymatic digestion. This includes cellulose, noncellulosic polysaccharides such as hemicellulose, pectic substances, gums, mucilages, and a non-carbohydrate component lignin, that are collectively known a dietary fiber $[54,55]$. These dietary fibers are important for digestive function, particularly regulating the traffic of feces through the digestive system which results in several important health effects. They include reduced risk for obesity, diabetes, and hypertension which was shown by the evidence that diets rich in fiber such as cereals, nuts, fruits, and vegetables are associated with a lower incidence of several diseases [54]. Evidence also shows that dietary fibers correlate with a lower incidence of colorectal cancers because they are able to trap mutagenic substances and decrease intestinal transit and consequently the time that these substances can be harmful to our cells (reviewed in [54]).

\subsection{Bioavailability of Bioactive Compounds}

Bioactive compounds are known to modulate the metabolic processes, through their antioxidant, antimicrobial, anticancer, or anti-inflammatory activities. These functions vary with the metabolic pathway and bioavailability in the body, as well as chemical structure and amount of dosage [3,9]. This means that bioactive compounds need to be bioavailable to exert beneficial health effects. Bioavailability can be defined as the fraction of a given food that the body can utilize, providing nutritional efficacy [56]. This is a crucial point to ensure the efficacy of bioactive compounds as limited bioavailability hinders the use of bioactive compounds as functional ingredients [9,57]. In this context, bioaccessibility, the fraction of a compound available for intestinal absorption [58], depends on several factors, including solubility, transporters, molecular structures, and metabolizing enzymes [9,59]. Bioaccessibility is also influenced by the composition of the food matrix and its physicochemical properties, such as $\mathrm{pH}$, temperature, and texture of the matrix [56]. The caloric content and the volume of the food matrix, for instance, can cause physiological changes in the gastrointestinal tract, affecting the bioaccessibility of digested compounds [56]. The different mechanisms of absorption are another factor that influences the bioavailability of 
food components. The absorption of bioactive compounds can be influenced by the interaction with other dietary ingredients, molecular transformations, transporters, metabolism, gut microbiota, and the solubility of lipophilic and hydrophilic compounds [35]. The molecular structure of a bioactive compound and its isomeric configuration can also affect its absorption considerably. Furthermore, the rate and extent of absorption can vary widely between individuals, depending on key factors, such as diet, genetic background, the composition of gut microbiota, and physical activity. All these factors result in changes to bioavailability [9].

The complex nature of bioactive compounds also has an important role in the bioavailability of food components [35]. For instance, the physiological activity of polyphenols does not depend directly on their prevalence in the human diet [35]. These compounds are, in fact, relatively poorly absorbed ( $0.3 \%$ to $43 \%)$ [60], thus limiting their biological effect on the body. The low intestinal absorption of polyphenols is determined by their chemical structure, while molecular weight, glycosylation, and esterification determine their bioavailability [61]. The source of food determines the form in which polyphenolic compounds occur [35]. Many phenolic aglycones are hydrophilic and can be absorbed through biological membranes by diffusion. However, most polyphenols occur in the glycosidic form, affecting their absorption [35]. Phenolic acids from the hydroxybenzoic family occur in low concentrations in food and it has been demonstrated that gallic acid is very well absorbed in the stomach, small intestine, or both [62]. The hydroxycinnamic acids (e.g., caffeic, ferulic, synaptic, or p-coumaric acids) often occur in their esterified form with quinic and tartaric acids or carbohydrate derivatives, being absorbed in the colon $[61,63]$. When ingested in their free form, they are rapidly absorbed from the stomach or small intestine [35]. Regarding flavonoids, the type of the substituted sugar moiety is an important factor and most flavonoids, except catechins, occur naturally in plants in conjunction with $\beta$-glycoside sugars [64]. The first step in the metabolism of flavonoids is the hydrolysis of the glycosidic bond, which may take place in the lumen of the intestine or enterocytes directly before absorption. Moreover, the efficiency of absorption is reduced when bacterial microflora is involved in the flavonoid metabolism and some studies have proved that the activity of microflora can make certain metabolites more active than their precursors [65]. Another important factor is the absence of a free hydroxy group at positions 5, 7, or 4, which protects the compounds from degradation [64]. Isoflavones and gallic acid, followed by catechin, quercetin glycosides, and flavanones, are best absorbed in the gastrointestinal tract. Anthocyanins can be absorbed in the glycosidic form and after hydrolysis by intestinal microflora. However, these compounds have shown the weakest absorption $[35,66]$. The best-known representative of stilbenes, resveratrol, is absorbed by passive diffusion or formation of complexes with membrane transporters. In the bloodstream, this compound goes through modifications by enterocytes and hepatocytes, and has been found in the free form, as glucuronide or sulfate derivatives. As for phytosterols, these are poorly absorbed and are excreted faster by the liver than cholesterol, explaining their low abundance in human tissues [20]. Vitamins have a low bioavailability due to low absorption, solubility, or conversion into inactive forms. The bioaccessibility of vitamins is also low and depends on their chemical structure, source, presence of elements acting synergistically or antagonistically, and mechanisms of intestinal absorption [35,67].

\subsection{Changes in Food Caused by Cooking and Other Processing Treatments}

Food cooking is intrinsically linked to human evolution because it enabled the inclusion of a myriad of nutrients in our diet that are not available in raw foods. Food cooking enables starch gelatinization and protein and antinutrient denaturation that are essential to improve digestibility and nutrient bioavailability to our cells [68]. Moreover, cooked diets improve the energy yield by delivering more energy and reducing the energetic costs of detoxification and defense against pathogens [68]. However, specifically considering the bioactive composition of cooked foods, particularly those of vegetal origins such as grains, tubers, legumes, and fruits, cooking causes a thermal degradation, which may reduce the 
concentration of certain phytochemicals. However, the process also favors matrix softening, improving the extractability of many phytochemicals and so, depending on the bioactive compound and the matrix where it exists, a net increase in their levels in the final cooked product often occurs [69]. This yield can be further improved by adjusting the cooking procedure to the properties of the target bioactive compounds. Steaming, for instance, is a milder process than boiling because the plant tissues are not in direct contact with the boiling liquid, usually water. Therefore, fewer soluble bioactive compounds are leached, and thermal degradation is not so extensive as in the boiling procedure. Accordingly, steaming will enrich the cooked food with phenols and glucosinolates, for instance, while it is too mild to solubilize carotenoids that require more aggressive cooking procedures [69]. Another class of important bioactive compounds, polyamides, which are present in many foods of plant origin, including green peppers, peas, citrus fruit, and broad beans, are drastically reduced by more than $60 \%$ by boiling and grilling but remain almost unaffected by microwave and sous vide cooking [70]. Vacuum cooking is gaining popularity due to its ability to preserve or even enhance the sensory and health properties of different foods. Using pumpkin cubes as a model food, Rinaldi, et al. [71] showed that vacuum cooking was more aggressive than sous vide or steaming, resulting in faster and deeper texture softening, but simultaneously less impact on pumpkin color despite the greatest extraction of some classes of carotenoids from the broken cells. In contrast, significant polyphenol extraction, especially of gallic acid and naringenin, was more efficient with sous vide and steaming pumpkins [71]. On this point, it is important to note that cooking can be used to add bioactive compounds to foods. To achieve this, very recently Gabrielle Alves de Carvalho, et al. [72] showed that French fries were enriched with virgin olive oil (VOO) bioactive compounds, namely sterols, upon deep-frying with VOO.

Fermentation was one of the first processes humans used to prepare and preserve food for periods in which nutrient availability could be scarce [73]. It is estimated that fermented foods constitute about one-third of the foods consumed daily worldwide [74]. This includes fermented vegetable and fruit foods and beverages with high nutritional value and bioactive composition, as well as a large range of prebiotic compounds that can stimulate the growth of beneficial bacteria in the human gut [74]. Fermentation is so popular because the extraction of bioactive compounds from raw foods is accompanied by unique textures and flavors widely appreciated in diverse diets around the world. Wine, beer, and cider are popular beverages obtained from the alcoholic fermentation of fruits while lactic fermentation is more popular with vegetables. This is the case for sauerkraut (fermented raw cabbage), tempeh (traditional Indonesian fermented soybeans), natto and miso (traditional Japanese fermented soybeans), kombucha (fermented tea leaves), and kimchi (traditional Korean fermented vegetables). Kimchi is made with several vegetables, especially cruciferous vegetables, and sub-ingredients of garlic, ginger, and red pepper powders, resulting in a bioactive-rich food with reported health benefits [75,76]. Another type of fermentation, the acetic fermentation of different fruits to obtain vinegar, is also widely used, particularly as a condiment to salads.

Drying food products is a preservation method traditionally used in many places in the world for foods such as grapes, tomatoes, figs, beans, corn, etc. By decreasing the moisture, fruits become less prone to microbial contamination and deterioration and can be stored for longer periods. Moreover, most constituents of the fruit become more concentrated, reinforcing new flavors and textures widely appreciated by consumers. Ultimately, drying can be used to obtain flours from diverse sources beyond the traditionally used cereals and grains. This includes flours obtained from apples, potatoes, sweet potatoes, carob, etc.

The release of bioactive compounds from the food matrices, making them accessible for further processing by the gut microbiota during digestion and eventually becoming absorbed into the bloodstream, is very important. This, however, is not a guarantee of bioactivity [77]. Once internalized to the bloodstream, other factors, which are out of the scope of this review, may modulate the availability of the bioactive compounds to the target cells. Nevertheless, food cooking and processing induce chemical and physical 
modifications that are essential to promote bioactive compounds' bioaccessibility and bioavailability [78-80].

\section{Nutritional and Bioactive Potential of Fresh Fruit and Vegetable Waste}

According to the most recent data available, approximately half of fruits and vegetables produced worldwide are wasted. This represents around 1.3 billion tons of wasted foods and constitutes the second highest cause of greenhouse gas emission, representing the emission of around 4.4 gigatons of greenhouse gas [7]. However, beyond the obvious environmental impact, this means that millions of tons of nutrients present in this food waste are being lost. Additionally, this is happening on the same planet where currently more than 500 million people do not have access to enough food to live, and the problem will be exacerbated as the world population continues to grow exponentially [7]. It is, therefore, imperative that each consumer contributes to wasting less food and part of that objective can be easily achieved just by integrating into our diet fruits and vegetables that we wrongly considered as inedible. The list of fruits and vegetables used in the human diet is potentially endless and so this review is focused on those with higher production and impact in terms of waste being discarded that can and should be further used in our diet. Their nutritional and bioactive potential is reviewed below in detail and summarized in Table 1. It should be noted, however, that this behavioral change should be accompanied by due diligence regarding food safety. Fruit and vegetable peels are often loaded with microorganisms and agrochemicals that should be thoroughly removed to maintain our safety.

Table 1. Main fruits and vegetables consumed worldwide with a rich bioactive waste composition.

\begin{tabular}{|c|c|c|c|c|c|}
\hline $\begin{array}{l}\text { Fruit or } \\
\text { Vegetable }\end{array}$ & $\begin{array}{l}\text { Edible Part } \\
\text { Composition: } \\
\text { Nutritional and } \\
\text { Bioactive Value }\end{array}$ & $\begin{array}{l}\text { Discarded Part } \\
\text { Composition: } \\
\text { Nutritional and } \\
\text { Bioactive Value }\end{array}$ & $\begin{array}{l}\text { Possible Dietary } \\
\text { Uses }\end{array}$ & Preparation & Ref. \\
\hline \multicolumn{6}{|l|}{ Fruit } \\
\hline Apple & $\begin{array}{l}\text { flesh: flavan-3-ols, } \\
\text { phenolic acids, flavonols, } \\
\text { dihydrochalcones, } \\
\text { anthocyanins, and } \\
\text { ascorbic acid }\end{array}$ & $\begin{array}{l}\text { peel: phenolic } \\
\text { compounds } \\
\text { (chlorogenic acid, } \\
\text { procyanidin B2, } \\
\text { epicatechin) }\end{array}$ & $\begin{array}{ll}- & \text { edible } \\
- & \text { vinegar } \\
- & \text { cider }\end{array}$ & 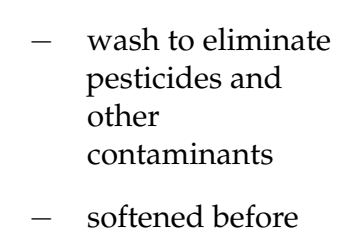 & [81-83] \\
\hline \multirow[t]{2}{*}{ Banana } & $\begin{array}{l}\text { flesh: fiber, } \\
\text { carbohydrates, } \\
\text { phenolics, biogenic } \\
\text { amines, phytosterols, } \\
\text { minerals; low-fat content }\end{array}$ & $\begin{array}{l}\text { peels: crude fiber, } \\
\text { carbohydrates, } \\
\text { crude protein, } \\
\text { phenolics, } \\
\text { minerals }\end{array}$ & $\begin{array}{ll}- & \text { edible after } \\
& \text { cooking } \\
- & \text { banana peel } \\
& \text { flour } \\
- & \text { fermented } \\
& \text { beverages }\end{array}$ & $\begin{array}{l}\text { ingestion } \\
\text { (cooked) } \\
-\quad \text { washed to } \\
\text { eliminate } \\
\text { pesticides and } \\
\text { other } \\
\text { contaminants }\end{array}$ & [8,84-86] \\
\hline & & $\begin{array}{l}\text { - blossoms: } \\
\text { saponins, } \\
\text { flavonoids, } \\
\text { glycosides, tannins, } \\
\text { steroids, phenols, } \\
\text { minerals, vitamins }\end{array}$ & $\begin{array}{l}-\quad \text { edible after } \\
\text { cooking }\end{array}$ & 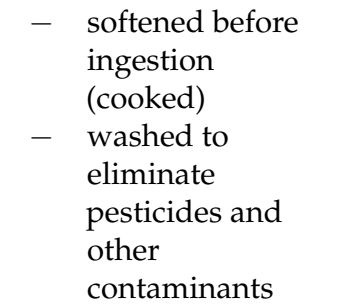 & [87] \\
\hline
\end{tabular}


Table 1. Cont.

\begin{tabular}{|c|c|c|c|c|c|}
\hline $\begin{array}{l}\text { Fruit or } \\
\text { Vegetable }\end{array}$ & $\begin{array}{l}\text { Edible Part } \\
\text { Composition: } \\
\text { Nutritional and } \\
\text { Bioactive Value }\end{array}$ & $\begin{array}{l}\text { Discarded Part } \\
\text { Composition: } \\
\text { Nutritional and } \\
\text { Bioactive Value }\end{array}$ & $\begin{array}{l}\text { Possible Dietary } \\
\text { Uses }\end{array}$ & Preparation & Ref. \\
\hline Citrus & $\begin{array}{l}\text { flesh: carbohydrates, } \\
\text { amino acids, } \\
\text { polyphenols, flavonoids, } \\
\text { vitamin } C \text {, minerals }\end{array}$ & $\begin{array}{l}\text { peel: fiber, } \\
\text { polyphenols, } \\
\text { flavonoids, } \\
\text { carotenoids, } \\
\text { vitamin C, } \\
\text { limonoids, } \\
\text { essential oils }\end{array}$ & $\begin{array}{ll}- & \text { citrus peel } \\
& \text { powder to } \\
& \text { flavor tea } \\
- & \text { natural food } \\
& \text { additive } \\
- & \text { fermented } \\
& \text { beverages } \\
- & \text { edible }\end{array}$ & $\begin{array}{ll}- & \text { washed to } \\
\text { eliminate } \\
\text { pesticides and } \\
\text { other } \\
\text { contaminants } \\
-\quad \text { softened before } \\
\text { ingestion } \\
\text { (cooked) }\end{array}$ & $\begin{array}{l}{[86,88-} \\
90]\end{array}$ \\
\hline Grape & $\begin{array}{l}\text { flesh: carbohydrates, } \\
\text { organic acids, } \\
\text { terpenoids, vitamins, } \\
\text { minerals, polyphenols }\end{array}$ & $\begin{array}{l}\text { - skin: phenolic } \\
\text { acids, stilbenes, } \\
\text { flavanols, } \\
\text { flavonols, } \\
\text { anthocyanins, } \\
\text { polysaccharides, } \\
\text { dietary fiber }\end{array}$ & $\begin{array}{ll}- & \text { grape } \\
& \text { pomace flour } \\
- & \text { natural food } \\
& \text { additive } \\
- & \text { fermented } \\
& \text { beverages }\end{array}$ & $\begin{array}{l}\text { - } \begin{array}{l}\text { washed to } \\
\text { eliminate }\end{array} \\
\text { pesticides and } \\
\text { other } \\
\text { contaminants }\end{array}$ & [91-98] \\
\hline & & $\begin{array}{l}\text { - } \text { seeds: unsaturated } \\
\text { fatty acids, vitamin } \\
\text { E, sterols, minerals }\end{array}$ & $\begin{array}{l}-\quad \text { grape seed } \\
\text { oil } \\
-\quad \begin{array}{l}\text { grape seed } \\
\text { flour }\end{array}\end{array}$ & & \\
\hline Kiwi fruit & $\begin{array}{l}\text { flesh: dietary fiber, } \\
\text { phenolic compounds, } \\
\text { vitamins, minerals }\end{array}$ & $\begin{array}{l}\text { - peel: rich in } \\
\text { carbohydrates, } \\
\text { dietary fiber, lipids, } \\
\text { protein, phenolic } \\
\text { compounds, } \\
\text { vitamins } C \text { and } E\end{array}$ & $\begin{array}{ll}- & \text { edible } \\
- & \text { natural } \\
& \text { additives in } \\
& \text { foods }\end{array}$ & $\begin{array}{l}\text { - washed to } \\
\text { eliminate } \\
\text { pesticides and } \\
\text { other } \\
\text { contaminants }\end{array}$ & [99-101] \\
\hline \multirow[t]{2}{*}{ Mango } & $\begin{array}{l}\text { flesh: rich in dietary } \\
\text { fiber, carbohydrates, } \\
\text { proteins, fats, and } \\
\text { phenolic compounds }\end{array}$ & $\begin{array}{l}\text { peel: rich in } \\
\text { dietary fiber, } \\
\text { phenolic } \\
\text { compounds, } \\
\text { carotenoids, } \\
\text { vitamins } C \text { and } \mathrm{E}, \\
\text { minerals }\end{array}$ & $\begin{array}{ll}- & \text { mango peel } \\
& \text { flour } \\
-\quad & \text { fermented } \\
& \text { beverages }\end{array}$ & 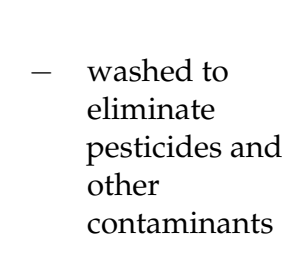 & $\begin{array}{l}{[86,102-} \\
106]\end{array}$ \\
\hline & & $\begin{array}{l}\text { - } \text { seeds: rich in } \\
\text { flavonoids, } \\
\text { phenolic acids, and } \\
\text { fats }\end{array}$ & $\begin{array}{l}\text { mango seed } \\
\text { edible fats }\end{array}$ & & \\
\hline Passion fruit & $\begin{array}{l}\text { flesh: vitamin A and C, } \\
\text { minerals, dietary fiber, } \\
\text { protein, phenolic } \\
\text { compounds }\end{array}$ & $\begin{array}{l}\text { - peel: fiber, pectin, } \\
\text { phenolic } \\
\text { compounds, and } \\
\text { minerals }\end{array}$ & $\begin{array}{ll}- & \text { passion fruit } \\
& \text { peel flour } \\
- & \text { natural food } \\
\text { additive }\end{array}$ & $\begin{array}{l}\text { - } \begin{array}{l}\text { washed to } \\
\text { eliminate }\end{array} \\
\text { pesticides and } \\
\text { other } \\
\text { contaminants }\end{array}$ & [107-115] \\
\hline & & - seeds: fats & - edible & & \\
\hline Pear & $\begin{array}{l}\text { flesh: sugars, vitamins, } \\
\text { organic and fatty acids, } \\
\text { amino acids, volatiles, } \\
\text { polyphenols, minerals }\end{array}$ & 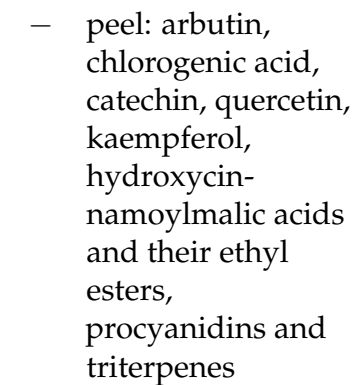 & - edible & $\begin{array}{l}\text { - washed to } \\
\text { eliminate } \\
\text { pesticides and } \\
\text { other } \\
\text { contaminants }\end{array}$ & {$[116,117]$} \\
\hline
\end{tabular}


Table 1. Cont.

\begin{tabular}{|c|c|c|c|c|c|}
\hline $\begin{array}{l}\text { Fruit or } \\
\text { Vegetable }\end{array}$ & $\begin{array}{l}\text { Edible Part } \\
\text { Composition: } \\
\text { Nutritional and } \\
\text { Bioactive Value }\end{array}$ & $\begin{array}{l}\text { Discarded Part } \\
\text { Composition: } \\
\text { Nutritional and } \\
\text { Bioactive Value }\end{array}$ & $\begin{array}{l}\text { Possible Dietary } \\
\text { Uses }\end{array}$ & Preparation & Ref. \\
\hline Peach & $\begin{array}{l}\text { flesh: dietary fiber, } \\
\text { minerals, sugars, } \\
\text { organic acids, phenolic } \\
\text { compounds, carotenoids, } \\
\text { volatiles }\end{array}$ & $\begin{array}{l}\text { peel: polyphenols } \\
\text { (flavanols, } \\
\text { hydroxybenzoic, } \\
\text { hydroxycinnamic } \\
\text { acids, flavonols), } \\
\text { carotenoids }\end{array}$ & - edible & $\begin{array}{l}\text { - washed to } \\
\text { eliminate } \\
\text { pesticides and } \\
\text { other } \\
\text { contaminants }\end{array}$ & [118-120] \\
\hline \multirow[t]{2}{*}{ Pineapple } & \multirow[t]{2}{*}{$\begin{array}{l}\text { flesh: carbohydrates, } \\
\text { dietary fiber, sugars, } \\
\text { organic acids, vitamins, } \\
\text { minerals }\end{array}$} & $\begin{array}{l}\text { - peel and rind: } \\
\text { carbohydrates, } \\
\text { proteins, pectin, } \\
\text { bromelain, } \\
\text { phenolic } \\
\text { compounds }\end{array}$ & $\begin{array}{ll}- & \text { vinegar } \\
& \text { production } \\
- & \text { tepache }(\mathrm{a} \\
& \text { fermented } \\
& \text { beverage }) \\
- & \text { flour } \\
& \text { production } \\
- & \text { natural food } \\
& \text { additive }\end{array}$ & 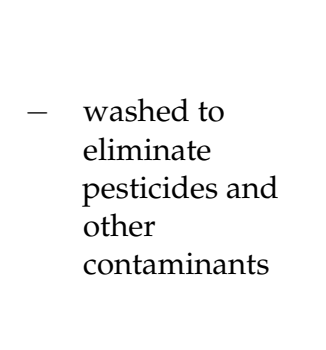 & \multirow[t]{2}{*}{$\begin{array}{l}{[73,121-} \\
123]\end{array}$} \\
\hline & & $\begin{array}{l}\text { - core: fiber, } \\
\text { bromelain, vitamin } \\
\text { C }\end{array}$ & - edible & $\begin{array}{l}-\quad \text { softened before } \\
\text { ingestion }\end{array}$ & \\
\hline \multirow[t]{2}{*}{ Plum } & \multirow[t]{2}{*}{$\begin{array}{l}\text { flesh: phenolic } \\
\text { compounds (chlorogenic } \\
\text { and gallic acids, } \\
\text { resorcinol, and rutin) } \\
\text { and ascorbic acid. }\end{array}$} & $\begin{array}{l}\text { - peel: polyphenols } \\
\text { (flavonoids, } \\
\text { anthocyanins), } \\
\text { ascorbic acid. }\end{array}$ & - edible & $\begin{array}{l}\text { - washed to } \\
\text { eliminate } \\
\text { pesticides and } \\
\text { other } \\
\text { contaminants }\end{array}$ & [124-126] \\
\hline & & $\begin{array}{l}\text { - seeds: dietary fiber, } \\
\text { protein, fat, } \\
\text { cucurbitin, } \\
\text { minerals, } \\
\text { tocopherol. }\end{array}$ & $\begin{array}{ll}- & \text { edible } \\
- & \text { natural food } \\
& \text { additive }\end{array}$ & $\begin{array}{l}\text { - } \text { external layer } \\
\text { must be softened } \\
\text { before ingestion } \\
\text { (cooking) }\end{array}$ & [127-134] \\
\hline \multirow[t]{2}{*}{ Watermelon } & $\begin{array}{l}\text { flesh: glycosides, } \\
\text { carotenoids, flavonoids, } \\
\text { alkaloids, carbohydrates, } \\
\text { fatty acids, essential oils. }\end{array}$ & $\begin{array}{l}\text { - } \text { rind: phenolic } \\
\text { compounds, } \\
\text { citrulline, pectin. }\end{array}$ & $\begin{array}{l}-\quad \text { natural food } \\
\text { additive }\end{array}$ & & \multirow[t]{2}{*}{ [135-139] } \\
\hline & & $\begin{array}{l}\text { - seeds: dietary oil, } \\
\text { minerals, vitamins, } \\
\text { phenolic } \\
\text { compounds }\end{array}$ & $\begin{array}{l}\text { - } \quad \text { watermelon } \\
\text { seed oil }\end{array}$ & & \\
\hline \multicolumn{6}{|l|}{ Vegetables } \\
\hline Broccoli & $\begin{array}{l}\text { florets: minerals, } \\
\text { vitamins, phenolic, } \\
\text { flavonoid compounds }\end{array}$ & $\begin{array}{l}\text { - stalks: phenolic } \\
\text { compounds, } \\
\text { sugars, pectic } \\
\text { polysaccharides, } \\
\text { free amino acids, } \\
\text { glucosinolates, } \\
\text { pectin }\end{array}$ & $\begin{array}{ll}- & \text { edible after } \\
& \text { cooking } \\
- & \text { natural food } \\
\text { additives } \\
\text { (soups, } \\
\text { flours, tea, } \\
\text { pasta) }\end{array}$ & 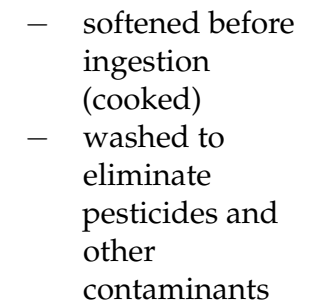 & [140-142] \\
\hline
\end{tabular}


Table 1. Cont.

\begin{tabular}{|c|c|c|c|c|c|}
\hline $\begin{array}{l}\text { Fruit or } \\
\text { Vegetable }\end{array}$ & $\begin{array}{l}\text { Edible Part } \\
\text { Composition: } \\
\text { Nutritional and } \\
\text { Bioactive Value }\end{array}$ & $\begin{array}{l}\text { Discarded Part } \\
\text { Composition: } \\
\text { Nutritional and } \\
\text { Bioactive Value }\end{array}$ & $\begin{array}{l}\text { Possible Dietary } \\
\text { Uses }\end{array}$ & Preparation & Ref. \\
\hline Cauliflower & $\begin{array}{l}\text { florets: fiber, minerals, } \\
\text { phenolics, ascorbic acid, } \\
\text { carotenoids, } \\
\text { glucosinolates }\end{array}$ & $\begin{array}{l}\text { - stems and leaves: } \\
\text { carbohydrates, } \\
\text { protein, fat, } \\
\text { glucosinolates } \\
\text { dietary fiber, } \\
\text { minerals }\end{array}$ & $\begin{aligned}- & \text { edible after } \\
& \text { cooking } \\
- & \text { leaf powder } \\
& \text { (food } \\
& \text { supplement) } \\
- & \text { natural food } \\
& \text { additive }\end{aligned}$ & $\begin{array}{l}- \\
\text { softened before } \\
\text { ingestion } \\
\text { (cooked) } \\
-\quad \text { washed to } \\
\text { eliminate } \\
\text { pesticides and } \\
\text { other } \\
\text { contaminants }\end{array}$ & [143-145] \\
\hline Potato & $\begin{array}{l}\text { flesh: carbohydrates, } \\
\text { polyphenols, vitamins, } \\
\text { and minerals }\end{array}$ & $\begin{array}{l}\text { peel: } \\
\text { glycoalkaloids, } \\
\text { polyphenols. } \\
\text { non-starch } \\
\text { polysaccharides, } \\
\text { proteins, lipids, } \\
\text { lignin, cellulose }\end{array}$ & $\begin{aligned}- & \text { edible after } \\
& \text { cooking } \\
- & \text { natural food } \\
& \text { additive }\end{aligned}$ & $\begin{array}{l}- \\
\text { ingestion } \\
\text { (cooking) } \\
-\quad \text { washed to } \\
\text { eliminate } \\
\text { pesticides and } \\
\text { other } \\
\text { contaminants }\end{array}$ & [146-150] \\
\hline Pumpkin & $\begin{array}{l}\text { flesh: dietary fiber, } \\
\text { carotenoids, phenolic } \\
\text { acids, flavonols, } \\
\text { minerals, vitamins }\end{array}$ & $\begin{array}{l}\text { - peel and rind: } \\
\text { dietary fiber } \\
\text { (pectins), } \\
\text { carotenoids, } \\
\text { phenolic acids, } \\
\text { flavonols, minerals, } \\
\text { vitamins }\end{array}$ & $\begin{array}{ll}- & \text { edible after } \\
& \text { cooking } \\
- & \text { natural food } \\
& \text { additive }\end{array}$ & 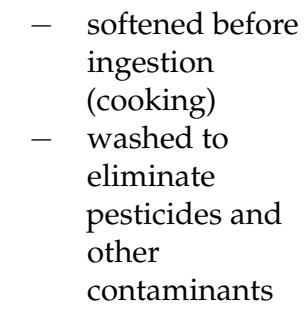 & {$[151,152]$} \\
\hline
\end{tabular}

\subsection{Fresh Fruits}

\subsubsection{Apple}

Apples are one of the most consumed fruits in Europe, and they are a great source of several bioactive compounds, especially phenolic compounds such as flavan-3-ols, phenolic acids, flavonols, dihydrochalcones, and anthocyanins. Furthermore, apples present a high content of ascorbic acid [81]. Apple waste is mainly composed of peels that are rich in bioactive compounds, in concentrations even higher than in the flesh. Apple peels are an important source of polyphenols, being the most abundant chlorogenic acid, procyanidin B2, and epicatechin [82].

\subsubsection{Banana}

The edible banana fruit (Musa spp.) is soft and sweet, and usually eaten raw. In contrast, plantain bananas (Musa $\times$ paradisiaca) have a firm and starchy pulp suitable for cooking as a vegetable. Both are seedless fruits, rich in carbohydrates, dietary fibers, certain vitamins, minerals (such as phosphorus, sodium, potassium, calcium, magnesium, iron, copper, zinc, and manganese), and several health-promoting bioactive phytochemicals [8]. Different studies have identified many of these bioactive compounds, such as carotenoids ( $\beta$-carotene), flavonoids, phenolics, biogenic amines, vitamins $\mathrm{A}, \mathrm{B}, \mathrm{C}$, and $\mathrm{E}$, and phytosterols $[8,84,153,154]$. Extracts from different parts of banana fruit and plants were shown to elicit effective protection against oxidative damage to cells and different human diseases triggered by these deleterious events, notably different forms of cancer (reviewed in [155]). These effects are possibly associated with the existence of specific phytoconstituents in banana plants and fruit, including ferulic acid, protocatechualdehyde, 2-pentanone, 4epicyclomusalenone, cycloeucalenol acetate, and chlorogenic acid, which have been shown to exhibit therapeutic cancer-preventing and anticancer abilities (reviewed in [155]). These bioactive compounds have been widely shown to have many positive effects on human 
health and banana has an effective antioxidant capacity higher than many berries [156]. This phytochemical composition certainly explains the efficiency of the traditional uses of bananas against degenerative diseases triggered by oxidative stresses [84]. Among the flavonoids found in bananas are quercetin, myricetin, kaempferol, and cyanidin $[8,28]$. Banana has been shown to contain a good amount of phytosterols, such as stigmasterol, $\beta$-sitosterol, campesterol, 24-methylene cycloartenol, cycloeucalenol, and cycloartenol, as well as a group of biogenic amines, the catecholamines [8]. In particular, the composition of the biogenic amines L-dopa and dopamine in banana makes this fruit very relevant as a supplement in the treatment of Parkinson's disease [153].

In contrast, and with exception of some producing countries in Asia and Latin America, banana peel and other parts of the fruit and plant are traditionally discarded in soil and used as compost to provide nutrients for the next harvests. However, banana peel is also rich in anthocyanins (delphinidin) and cyanidins [8], as well as in $\beta$-carotene, gallocatechin, and vitamins A, C, and E $[157,158]$. Additionally, it has a high content of dietary fibers, including hemicellulose and pectin polysaccharides [157]. Like the pulp, banana peels are also rich in phytosterols and catecholamines (biogenic amines). Borges, et al. [159] reported that banana peel showed superior phenolic compound and mineral levels compared to pulp. This composition of banana peels certainly explains the bioactive effects reported in traditional uses to promote wound healing, mainly from burns, and to help overcome or prevent several illnesses, such as depression [153].

The banana rhizome is very rich in phenolic compounds with important antioxidant activities [160]. The main phenolics found include ferulic, sinapic, salicylic, gallic, p-hydroxybenzoic, vanillic, syringic, gentisic, and p-coumaric acids, besides catechin, epicatechin, tannins, and anthocyanins [8]. Banana flowers or blossoms are also treated as agricultural waste when they are extirpated from the plant during the growing process, as they compete for the nutrients necessary for the growing fruit [161]. However, high amounts of different bioactive compounds, such as umbelliferone and lupeol, have been reported in ethanol extracts of banana flowers [162]. Part of these bioactive compounds has potential against cardiovascular diseases and diabetes. Evidence of such effects was provided by Wistar rats fed with an experimental diet incorporating banana blossom, which revealed modulation of hypocholesterolemic and hypoglycemic responses [155]. Recently, Sheng, et al. [163] also provided evidence that banana flower phytosterols, notably $\beta$-sitosterol, have the potential to prevent diseases associated with abnormal blood sugar and AGE levels, such as diabetes. Finally, extracts from the bracts of the banana flower were shown to contain anthocyanins (delphinidin, pelargonidin, peonidin, and malvidin), alkaloids, glucosides, flavonoid and phenolic compounds, saponins, terpenoids, coumarins, and steroids [161], as well as minerals $(\mathrm{K}, \mathrm{Cu}, \mathrm{Ca}, \mathrm{Fe}$, and $\mathrm{P})$ and vitamins $(\mathrm{A}, \mathrm{C}$, and $\mathrm{E})$ [87].

\subsubsection{Citrus Fruit}

Citrus fruits, mainly orange and lemon, are very popular worldwide. These fruits are rich in carotenoids, flavonoids, terpenes, limonoids, and many other bioactive compounds of nutritional and nutraceutical value, including polymethoxylated flavones (PMFs). These flavones were shown to elicit several bioactive effects against metabolic disorders, atherosclerosis, inflammation, neuroinflammation, cancer, and oxidation (reviewed in [164]). In addition, citrus essential oils are rich in limonoids and terpenes, which are widely appreciated for their antioxidant, antimicrobial, and flavoring properties with broad industrial applications [164]. Citrus peels are the main residue originating from citrus fruit, being very rich in dietary fibers (cellulose, hemicellulose, and pectin), bioactive compounds (phenolic compounds, flavonoids, and carotenoids), and vitamin C $[90,165,166]$. A myriad of bioactive compounds has been reported in citrus peels. A study recently conducted by Yaqoob, et al. [167], for instance, revealed that kinnow peel contains six flavones, eight favon-3-ols, five flavanones, four favan-3-ols, one anthocyanin, phenolic acids, and limonoids. Other reports point to citrus peels as promising sources of naringin, $\beta$-carotene, hesperidin, neohesperidin, and pectin $[168,169]$. Tangerines grown in Madeira, 
for instance, have a very intense and unique aroma which is mainly due to the presence of high amounts of volatile bioactive compounds in their peels, including thymol and dimethyl anthranilate [170]. Overall, citrus peels gained considerable attention due to their content in essential oils, mainly constituted by a mixture of volatile compounds such as terpenes (limonene) and oxygenated derivates such as aldehydes (citral), alcohols, and esters [89,169].

\subsubsection{Grape}

Grape is a fruit crop with a wide range of products, from fresh fruit and juice to other highly processed products, such as jelly and wine. Interest in these products is greatly related to the high content of phytonutrients belonging to numerous families, such as carbohydrates, organic acids, terpenoids, vitamins, and minerals, but most importantly polyphenols [94]. Studies have proved that these health-promoting compounds are highly accumulated in the skin, seeds, stems, and leaves of grapes [94,171]. Several studies have identified various phenolic compounds in grape skins, including phenolic acids, stilbenes, flavanols, flavonols, and anthocyanins [91,95,98], namely malvidin-3,5-di-O-glucoside and the flavonol quercetin- $\beta$-D-glucoside [172]. Regarding the nutritional value of grape pomace, it can be observed that red grape varieties are rich in dietary fibers, consisting mainly of lignin, cellulose, and hemicellulose [93]. Grape seeds' most relevant component is oil, which is rich in unsaturated fatty acids, such as linoleic and oleic acids, vitamin E, and sterols. Seeds also present significant amounts of essential minerals, predominantly $\mathrm{K}$, $\mathrm{Fe}$, and $\mathrm{Zn}[96]$.

\subsubsection{Kiwi Fruit}

Kiwi fruit is one of the most commercialized fruits in the international markets and is loaded with nutrients such as vitamins, minerals, and a myriad of bioactive compounds $[100,101]$. Its peel is a source of macronutrients, namely carbohydrates, dietary fiber, lipids, proteins, and vitamins C and E [99]. It also presents high biological activity due to the content in phenolic compounds, such as flavonoids, organic acids (quinic, malic, and malic), epicatechin, and anthocyanins [101]. Kiwi fruit skin has been reported to exert higher antioxidant, antibacterial, and anticancer activities than the pulp [173].

\subsubsection{Mango}

Mangoes are one of the most popular fruits worldwide, and their production causes high amounts of waste. Mango peels and seeds are the main byproducts produced, having a considerable concentration of bioactive compounds [104]. Mango peel is rich in vitamins $\mathrm{C}$ and $\mathrm{E}$, and in several minerals $(\mathrm{Ca}, \mathrm{K}, \mathrm{Mg}, \mathrm{Na}, \mathrm{Fe}, \mathrm{Mn}, \mathrm{Zn}$, and $\mathrm{Cu}$ ) [105], and contains pectins and anthocyanins [103] and a higher polyphenol content than its pulp [105]. Among the phytochemicals in mango peel reported in the literature, we can find mangiferin, benzophenone derivates, xanthones, phenolic acids, fatty acids, flavonoids, procyanidins, penta-O-galloyl-glucoside, methyl gallate, tetra-O-galloyl-glucoside, maclurin di-O-galloylglucoside, and isoquercitrin [106]. Mango seeds also have a very interesting bioactive composition which includes high concentrations of the major flavonoids and phenolic acids, gallic acid, catechin, chlorogenic acid, caffeic acid, ellagic acid, rutin, quercitrin, quercetin, and kaempferol [106]. Additionally, these seeds are a source of fats, being particularly rich in palmitic, stearic, oleic, and linoleic acids [102].

\subsubsection{Passion Fruit}

Passion fruit is a tropical fruit with worldwide popularity attributed to its appealing sensorial properties, and its processing for juice results in great quantities of byproducts [174]. Passion fruit peel comprehends about $52 \%$ of the total fruit weight and has been shown to have a high content of fiber [108], pectin [112], and phenolic compounds, predominantly isoorientin, and lower concentrations of other flavonoids, such as vicenin, isovitexin, vitexin, orientin, and schaftoside $[113,115]$. The peels have also been indicated 
as an excellent source of essential minerals, such as K and Ca [109]. The use of orange passion fruit peel flour in foods (bread, cakes, biscuits, and cereal bars) has likewise been reported to increase their nutritional qualities, contributing to the enrichment of the total dietary fiber, minerals, and bioactive compounds [114]. Besides the peels, passion fruit seeds are also a byproduct with great nutritional value, mainly due to their high lipid content, especially oleic and linoleic acids [109].

\subsubsection{Peach}

Peach is a fruit highly appreciated for its aromatic characteristics and its richness in dietary fiber, minerals, sugars, organic acids, phenolic compounds, carotenoids, and volatiles [119]. The peach peel contains plenty of polyphenols, such as flavanols, hydroxybenzoic and hydroxycinnamic acids, and flavonols [120]. Furthermore, high carotenoid content was observed in peach peel extracts [118].

\subsubsection{Pear}

Pear fruit is widely consumed worldwide and can be found in several processed products. It is famous for its many medical functions, such as antidiabetic, anticarcinogenic, and anti-inflammatory properties [117], and various nutrients and bioactive compounds, including sugars, vitamins, organic and fatty acids, amino acids, volatiles, polyphenols, and minerals are attributed to pear fruit [116]. A variety of bioactive compounds has been identified in pear peel, including arbutin, chlorogenic acid, catechin, quercetin, kaempferol, hydroxycinnamoylmalic acids and their ethyl esters, and procyanidins and triterpenes. These compounds were found in pear peel in concentrations approximately 6-20 times higher than those in the flesh of the pear [116].

\subsubsection{Pineapple}

Pineapple is another highly appreciated tropical fruit in the world due to its nutritional and organoleptic properties. In recent years, the pineapple market has grown significantly, generating huge volumes of byproducts (mainly the core and peel), which have negative economic and environmental impacts [121]. Pineapple core is a rich source of fibers, bromelain [123], and vitamin C [122]. The peel of pineapple contains carbohydrates, proteins, pectin, bromelain, and phenolic compounds, namely gallic acid, catechin, epicatechin, and ferulic acid [123].

\subsubsection{Plum}

Plum (Spondias spp.) has been widely used for medicinal and therapeutic purposes since it is rich in phenolic compounds, namely chlorogenic and gallic acids, resorcinol, and rutin [126]. Moreover, it is a source of ascorbic acid [125]. Plum peel is proved to have high amounts of polyphenols, including flavonoids and anthocyanins, and ascorbic acid [124].

\subsubsection{Watermelon}

Watermelon is the second-largest fruit crop worldwide and causes a great amount of biowaste, mainly the rind and seeds, that represent an environmental hazard [175]. Watermelon rind is an interesting natural source of citrulline, a non-protein $\alpha$-amino acid reported to have antioxidant and vasodilatation activity [176], and pectin, a polysaccharide widely used as an additive in the food industry [175]. Additionally, higher levels of phenolic compounds are found in watermelon rind than in its flesh [176]. In turn, watermelon seeds are an excellent source of dietary oils [135], with high levels of linoleic acid and lower concentrations of oleic, palmitic, and stearic acids, as well as minerals $(\mathrm{P}, \mathrm{K}, \mathrm{Na}$, and $\mathrm{Mg}$ ) [136]. Enemor, Oguazu, Odiakosa and Okafor [137] also reported a vast vitaminic repertoire in watermelon seeds, namely vitamins $\mathrm{A}$ and $\mathrm{C}$ and in minor concentrations B1, B2, B3, B6, B9, B12, D, E, and K. A myriad of other bioactive compounds has been also reported in these seeds, mainly sinapic acid, and lower amounts of other phenols, 
flavonoids, saponin, tannins, cardiac and cyanogenic glycosides, terpenoids, phytosterol, steroids, and phytates [138,177].

\subsection{Vegetables}

\subsubsection{Broccoli}

Broccoli is a Brassica vegetable widely consumed worldwide, owing to its healthpromoting properties $[143,178]$. About $60-75 \%$ of world broccoli production is wasted during harvesting [141], creating a large number of byproducts, most of which are edible [140]. Broccoli stalks, for instance, constitute an abundant source of pectin, also being rich in phenolic compounds, fructose, glucose, mannitol, polysaccharides, free amino acids, and glucosinolates [140,141].

\subsubsection{Cauliflower}

Cauliflower is another Brassica vegetable rich in natural antioxidants. It is known to have anticancerous properties due to its abundance in glucosinolates [143,178]. Cauliflower stems and leaves present carbohydrates, protein, fat, and glucosinolates [144]. Further, cauliflower leaves are very rich in dietary fiber and minerals [145].

\subsubsection{Potato}

Potato is the fourth main crop consumed worldwide and it is an important constituent in the human diet [148].

Potato peels represent about $10 \%$ of the total potato waste and are an inexpensive source of valuable bioactive compounds, such as secondary metabolites and cell wall materials, which can be used to functionalize foods or replace synthetic additives with natural ingredients $[179,180]$. Studies have shown that potato peels contain glycoalkaloids, such as $\alpha$-solanine and $\alpha$-chaconin, polysaccharides, the phenolic compounds quercetin and rutin, and ferulic, gallic, p-coumaric, caffeic, and chlorogenic acids [180,181], which are important precursors for steroid hormones and natural antioxidants, respectively [149]. Acylated anthocyanins were identified in the peels of red and purple potato varieties, with pelargonidin, peonidin, and malvidin being the most prominent aglycones [150].

\subsubsection{Pumpkin}

The pumpkin (Cucurbita spp.) is a well-known vegetable all over the world and is unquestionably a source of valuable nutrients, such as carotenoids [129]. Pumpkin seeds are rich in oil and several health-promoting compounds [128,132]. The oil extracted from pumpkin seed kernels is rich in protein and fat, including palmitic, stearic, oleic, and linoleic acids. However, the level of accumulation of these fatty acids differs greatly among pumpkin varieties [134]. The main proteins previously found in pumpkin seed consist of storage salt-soluble globulins (cucurbitin), albumins, glutelins, and prolamins [127]. According to the literature, pumpkin seed kernels also accumulate high levels of minerals such as $\mathrm{Fe}, \mathrm{Zn}, \mathrm{K}, \mathrm{Ca}, \mathrm{Mg}, \mathrm{Mn}, \mathrm{Cu}$, and Na, as well as tocopherol [133,134]. Pumpkin skins have broad variations in terms of composition and texture, but overall, they are edible and very rich in many bioactive compounds, notably high amounts of carotenoids and polyphenols with broad uses in food, pharmaceutical, and cosmetic industries. Bioactive colorant pigments and skin regeneration formulations are examples of promising applications for pumpkin peel extracts [151]

\subsection{Dietary Uses of Fruit and Vegetable Waste}

The inclusion in our diet of fruit and vegetable spare parts, often seeds and peels that are discarded and here generically considered as waste, is not new. Banana peels and blossoms, for instance, have been widely consumed for centuries as a curry, boiled, fried, etc. in Asia (Sri Lanka and India) and some countries in Latin (Brazil) and Central America (Caribbean islands) [155]. In Ethiopia, the Indian wild banana Ensete superbum (Cliff banana) is the main staple food because this variety is very resistant to severe drought. 
Moreover, its seeds have been used for generations to treat several health problems such as diabetes, leukorrhea, kidney stones, and dysuria [182]. Banana flowers, pseudostem, and unripe fruits are also consumed as vegetables by many indigenous communities [183], including rural peoples in India [184]. Despite the popularity of the fruit, banana peels are not used in the human diet in most developing countries. Recently, however, the growing concerns of consumers about the sustainability of the planet placed cooking banana peels under social media attention. Hopefully, Nigella Lawson's banana peel curry [185] will trigger a change in the way we consider fruit and vegetable waste in our diet. Similarly, banana blossoms seem to be a very versatile food to substitute meat proteins, taking into consideration the great number of different potential recipes such as curries, fish and chips, linguini, paella, salads, soups, fish pies, and crab cakes. Beyond the historic use of banana waste in the human diet, which would be the strongest argument that it is a safe procedure, evidence from different studies demonstrates the nutritional and bioactive value of such waste. Boiling bananas, often plantain or cooking banana varieties, increases the phenolic compound content of banana [159] because heat softens the vegetable structures, therefore releasing the bioactive compounds into the medium. In this case, banana peel prevents the loss of those bioactives in the water. Similar studies reported an increase in phenolic content during thermal processing of banana peel $[184,186]$.

For most people, the hairy skin of kiwi fruit is not very appellative to eat. However, not only are the peels edible, eating skin-on kiwi greatly increases nutrient intake, namely of fibers, vitamins $C$ and $E$, and a great number of bioactive compounds $[101,187]$. Consumption of whole SunGold kiwifruit (including the skin), for instance, was shown to increase the fiber, vitamin $\mathrm{E}$ and folate contents by 50,32 , and $34 \%$, respectively [188]. To facilitate the ingestion of kiwi skin, the hairs can be easily removed by rubbing the fruit, scrubbing it with a vegetable brush, or scraping it lightly with a spoon.

Tannins are widely present in many fruits, particularly in their wasted parts, such as citrus and banana peels. This abundance is the main reason why consumers reject those parts because tannins confer high astringency and unpleasant flavors. When present in lower amounts, however, tannins are more easily tolerated by consumers, as occurs with the proanthocyanidins present in grape skins. Different strategies, however, can be used to mitigate the high abundance of tannins, such as sweetening to obtain marmalades and jams, or fermentation to obtain beverages, such as tepache [73]. This is a traditional Mexican beverage that is obtained by fermentation of different fruits and fruit waste, according to the region where it is produced. Pineapple rinds and tamarind, banana peels, corn husks, crushed corn, persimmon, apple, jujube, pomegranate, plum, cranberry, sour cherry, soursop, mango, orange, and papaya are among the tepache varieties in traditional Mexican food folklore [73]. Although there are not many studies in the literature about these traditional fermented beverages, it is known that the use of fruit during the fermentation process of beverages such as beer enhances the content of bioactive compounds, in particular polyphenols and carotenoids [86]. Acetic fermentation to obtain vinegar is also a popular process to give additional uses to many fruit wastes [83]. Traditionally associated with wine, vinegar can be produced from many fruit wastes such as the peels and cores from apple, kiwi fruit, pineapple, pear, mango, melon, watermelon, etc. In simple terms, any fruit and its waste can be fermented to obtain vinegar enriched with different cocktails of nutrients and bioactive compounds. In this respect, Sandor Katz is carrying out very relevant work unveiling ancient techniques behind fermentations that everyone can use at home [189].

Cucurbita moschata Duchesne (squash and pumpkin varieties) is a plant food highly appreciated for the content of nutrients and bioactive compounds, including polyphenols and carotenoids. Its peel (including the rind layers immediately below the peel) are particularly rich in pectins which can be used in various food processing operations due to their gelling properties. Pectins are also used as thickening agents and stabilizers in jams, jellies, etc. [152]. Pumpkin peel therefore has many industrial applications, but it can also be very useful for consumers even with minimal cooking procedures. Although ripe pumpkin 
peel is very hard to eat, it can be easily softened by different cooking procedures, such as boiling, roasting, frying, or baking, for use in pies and cakes. Table 2 presents selected examples from the literature showing cooking procedures to incorporate fruit wastes in our diet.

Table 2. Examples of possible uses and cooking procedures for several fruit and vegetable wastes.

\begin{tabular}{|c|c|c|}
\hline Fruit or Vegetable Waste & Suggested Cooking Procedure & Ref. \\
\hline Apple peel & $\begin{array}{l}\text { Cookies: peels were blanched in hot water for } 30 \mathrm{~s} \text {, washed and dried }\left(48 \mathrm{~h}, 60^{\circ} \mathrm{C}\right) \text {, } \\
\text { ground, and used to make cookies. }\end{array}$ & [190] \\
\hline Citrus peel & $\begin{array}{l}\text { Jam: washed and crushed peels were mixed with water to form a puree, and then sugar, } \\
\text { pectin, and citric acid were added to obtain a jam-like texture. }\end{array}$ & [191] \\
\hline Passion fruit peel & $\begin{array}{l}\text { Cookies: peels were immersed in water }\left(100^{\circ} \mathrm{C}, 15 \mathrm{~min}\right) \text { and cooled on ice, epicarp was } \\
\text { separated with a spoon, dried }\left(60^{\circ} \mathrm{C}\right) \text {, ground, and used to make cookies. }\end{array}$ & [192] \\
\hline \multirow[t]{2}{*}{ Pineapple core } & Pineapple dried core: core was cut into small pieces and dried in a microwave oven. & [193] \\
\hline & Pineapple core fiber was extracted and incorporated into dough and steamed bean. & [194] \\
\hline \multirow[t]{2}{*}{ Pineapple peel } & $\begin{array}{l}\text { Beef marination: the peel was washed, cut, mashed with a blender, and filtered; the juice } \\
\text { was then used to marinate beef. }\end{array}$ & [195] \\
\hline & $\begin{array}{l}\text { Gluten-free muffins: peels were sanitized with } 100 \mathrm{ppm} \text { chlorinated solution for } 15 \mathrm{~min} \text {, } \\
\text { dried for } 8 \mathrm{~h} \text { at } 60^{\circ} \mathrm{C} \text {, and milled to obtain flour. }\end{array}$ & [196] \\
\hline \multirow[t]{2}{*}{ Plum peel } & $\begin{array}{l}\text { Marmalade: peels were mixed with water and filtered to make a puree, and then sugar } \\
\text { syrup, pectin, and citric acid were added to obtain marmalade. }\end{array}$ & [197] \\
\hline & $\begin{array}{l}\text { Jam: washed and crushed peels were mixed with water to form a puree, and then sugar, } \\
\text { pectin, and citric acid were added to obtain a jam-like texture. }\end{array}$ & [198] \\
\hline \multirow[t]{3}{*}{ Potato peel } & $\begin{array}{l}\text { Potato peel chips: peels were dried, milled, and sieved to obtain flour, then mixed with } \\
\text { wheat flour to make deep-fried chips. }\end{array}$ & [199] \\
\hline & $\begin{array}{l}\text { Potato peel dough: peels were dried at } 50^{\circ} \mathrm{C} \text { in an oven for } 48 \mathrm{~h} \text {, ground in a mixer } \\
\text { grinder to make flour, mixed with wheat flour, and incorporated into dough. }\end{array}$ & [200] \\
\hline & $\begin{array}{l}\text { Gluten-free bread: peels were sanitized with sodium hypochlorite solution, dried at } 70^{\circ} \mathrm{C} \\
\text { for } 24 \mathrm{~h} \text { in a forced-air oven, and ground using a blender; the flour obtained was used to } \\
\text { produce gluten-free bread. }\end{array}$ & [201] \\
\hline Pumpkin rind and seeds & $\begin{array}{l}\text { Bread: rinds and seeds were dried in an oven at } 60^{\circ} \mathrm{C} \text { for } 24 \mathrm{~h} \text { and ground using a grinder } \\
\text { to obtain a fine particle size, then the flour obtained was incorporated into bread. }\end{array}$ & [202] \\
\hline \multirow[t]{2}{*}{ Watermelon rind } & $\begin{array}{l}\text { Marmalade: rind was mixed with water and filtered to make a puree, and then sugar } \\
\text { syrup, pectin, and citric acid were added to obtain marmalade. }\end{array}$ & [203] \\
\hline & $\begin{array}{l}\text { Watermelon rind flour: rind was cut into small chips, dried at } 60^{\circ} \mathrm{C} \text { for } 48 \mathrm{~h} \text {, and milled } \\
\text { into powdered flour to use in cakes. }\end{array}$ & [204] \\
\hline Watermelon seeds & $\begin{array}{l}\text { Watermelon snacks: seeds were ground in a mixer grinder into a fine powder and sieved } \\
\text { to obtain uniform size distribution of the grains; the flour obtained was mixed with rice } \\
\text { and corn flour and used to make snacks. }\end{array}$ & [205] \\
\hline
\end{tabular}

Many more examples of home food applications and recipes for fruit and vegetable waste could be included in this review. This would be, however, a very long list that obviously cannot be included here. Instead, the selected examples constitute a flavor of the bioactive potential that is being neglected in the fruit and vegetable waste that is discarded in our kitchens (Figure 3). 


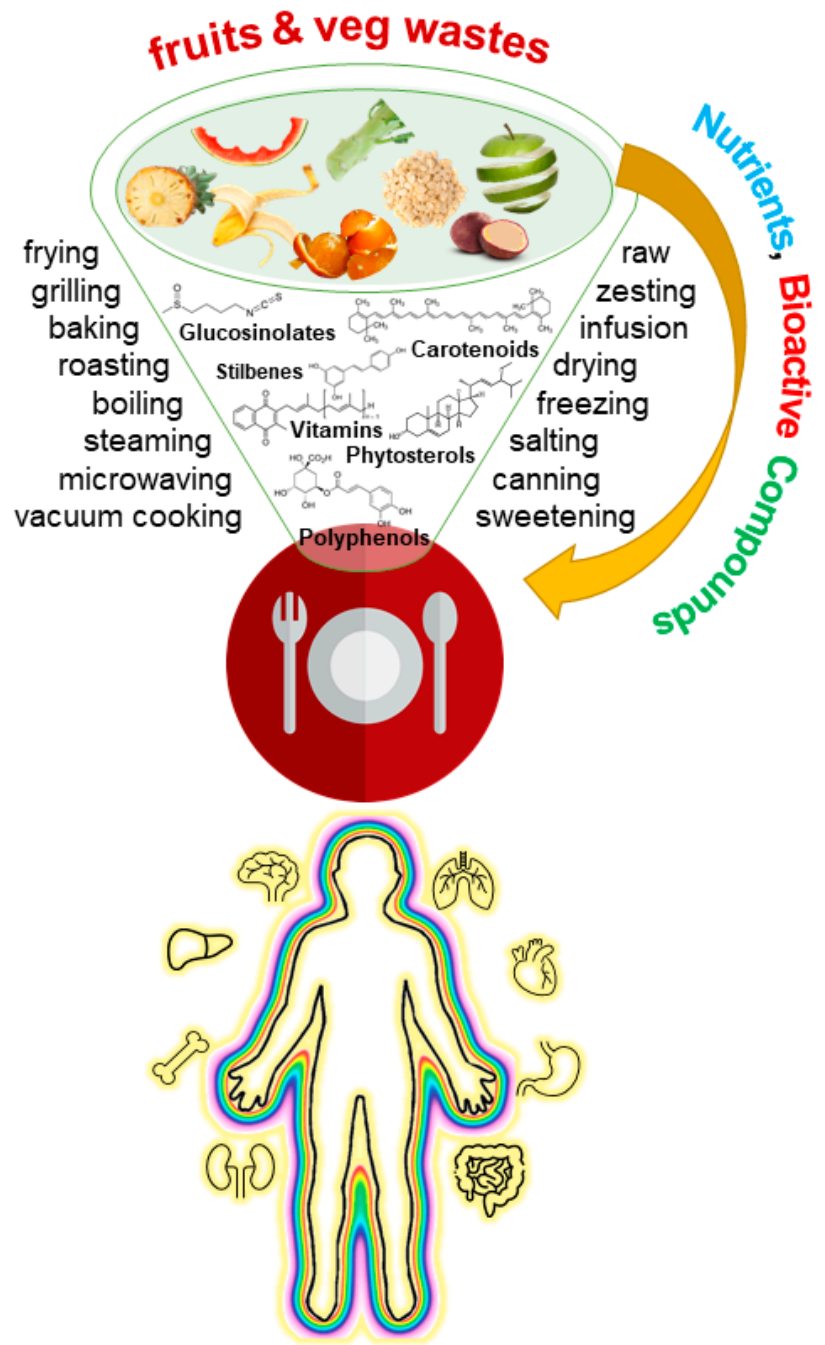

Figure 3. Integrative overview of the rationale behind fruit and vegetable waste cooking to include important molecules with reported bioactive effects in our diet.

\section{Concluding Remarks}

Despite the global climate emergency and enormous challenges to produce more food and better food for an exponentially growing population, fruit and vegetable waste in our homes caused by inadequate cooking and eating habits is absurdly high, having a great impact on greenhouse gas emissions. This review aggregated evidence of the high bioactive potential of spare parts of fruits and vegetables, particularly peels, rinds, seeds, and cores, that are ubiquitous in our kitchens, and we must start looking to them as food and not waste. Many of these behaviors to include in our diet what we always consider as waste are not new, instead, they have been practiced for centuries in many parts of the world. This constitutes a cultural dietary heritage that we must rediscover to benefit our health and a sustainable future for the planet.

Author Contributions: J.A.M.P. planned and managed the manuscript; J.A.M.P. and J.S.C. were responsible for Section 1; J.S.C. was responsible for Section 2, J.A.M.P., C.V.B. and C.F.P.A. collaborated on Section 3, J.A.M.P. and C.F.P.A. collaborated on Section 4; J.A.M.P. was responsible for Section 5; J.A.M.P. and J.S.C. edited the manuscript; J.A.M.P. made its submission. All authors have read and agreed to the published version of the manuscript.

Funding: This research was funded by Fundação para a Ciência e a Tecnologia (FCT) through the CQM Base Fund, UIDB/00674/2020, the Programmatic Fund, UIDP/00674/2020, Madeira 14-20 Program, project PROEQUIPRAM, Reforço do Investimento em Equipamentos e Infraestruturas Científicas RAM (M1420-01-0145-FEDER-000008), Agência Regional para o Desenvolvimento da 
Investigação Tecnologia e Inovação (ARDITI) through the project M1420-01-0145-FEDER-000005Centro de Química da Madeira-CQM + (Madeira 14-20 Program) and Project M1420-09-5369-FSE000001 for the post-doctoral fellowship granted to J.A.M.P. Núcleo Regional da Madeira da Liga Portuguesa Contra o Cancro (NRM-LPCC) is acknowledged for the professional internship granted to C.V.B.

Institutional Review Board Statement: Not applicable.

Informed Consent Statement: Not applicable.

Data Availability Statement: Not applicable.

Conflicts of Interest: The authors declare no conflict of interest.

\begin{abstract}
Abbreviations
AD—Alzheimer's disease, CVDs—cardiovascular diseases, EU-European Union, NDDsneurodegenerative diseases, ODs—oncologic diseases, ROS—reactive oxygen species, US-United States of America, VOO—virgin olive oil.
\end{abstract}

\title{
References
}

1. United Nations Department of Economic and Social Affairs. World Population Prospects 2019: Highlights; United Nations Department of Public Information: New York, NY, USA, 2019.

2. European Commission, Directorate-General for Research and Innovation. A sustainable bioeconomy for Europe: Strengthening the Connection between Economy, Society and the Environment: Updated Bioeconomy Strategy, Publications Office. 2018. Available online: https:/ / data.europa.eu/doi/10.2777/478385 (accessed on 10 February 2022).

3. Saini, A.; Panesar, P.S.; Bera, M.B. Valorization of fruits and vegetables waste through green extraction of bioactive compounds and their nanoemulsions-based delivery system. Bioresour. Bioprocess. 2019, 6, 26. [CrossRef]

4. Jiménez-Moreno, N.; Esparza, I.; Bimbela, F.; Gandía, L.M.; Ancín-Azpilicueta, C. Valorization of selected fruit and vegetable wastes as bioactive compounds: Opportunities and challenges. Crit. Rev. Environ. Sci. Technol. 2019, 50, 2061-2108. [CrossRef]

5. Genkinger, J.M.; Platz, E.A.; Hoffman, S.C.; Comstock, G.W.; Helzlsouer, K.J. Fruit, vegetable, and antioxidant intake and all-cause, cancer, and cardiovascular disease mortality in a community-dwelling population in Washington County, Maryland. Am. J. Epidemiol. 2004, 160, 1223-1233. [CrossRef] [PubMed]

6. Freedman, N.D.; Park, Y.; Subar, A.F.; Hollenbeck, A.R.; Leitzmann, M.F.; Schatzkin, A.; Abnet, C.C. Fruit and vegetable intake and head and neck cancer risk in a large United States prospective cohort study. Int. J. Cancer 2008, 122, 2330-2336. [CrossRef]

7. FAO. FAO Statistical Yearbook 2021-World Food and Agriculture; FAO: Rome, Italy, 2021. [CrossRef]

8. Sidhu, J.S.; Zafar, T.A. Bioactive compounds in banana fruits and their health benefits. Food Qual. Saf. 2018, 2, 183-188. [CrossRef]

9. Rein, M.J.; Renouf, M.; Cruz-Hernandez, C.; Actis-Goretta, L.; Thakkar, S.K.; da Silva Pinto, M. Bioavailability of bioactive food compounds: A challenging journey to bioefficacy. Br. J. Clin. Pharmacol. 2013, 75, 588-602. [CrossRef]

10. Yahia, E.M.; García-Solís, P.; Celis, M.E.M. Chapter 2-Contribution of Fruits and Vegetables to Human Nutrition and Health. In Postharvest Physiology and Biochemistry of Fruits and Vegetables; Yahia, E.M., Ed.; Woodhead Publishing: Sawston, UK, 2019; pp. 19-45. [CrossRef]

11. Islam, M.A.; Khandker, S.S.; Alam, F.; Khalil, M.I.; Kamal, M.A.; Gan, S.H. Alzheimer's Disease and Natural Products: Future Regimens Emerging from Nature. Curr. Top. Med. Chem. 2017, 17, 1408-1428. [CrossRef]

12. Crous-Bou, M.; Minguillon, C.; Gramunt, N.; Molinuevo, J.L. Alzheimer's disease prevention: From risk factors to early intervention. Alzheimers Res. 2017, 9, 71. [CrossRef]

13. Lapuente, M.; Estruch, R.; Shahbaz, M.; Casas, R. Relation of Fruits and Vegetables with Major Cardiometabolic Risk Factors, Markers of Oxidation, and Inflammation. Nutrients 2019, 11, 2381. [CrossRef]

14. Sharifi-Rad, J.; Rodrigues, C.F.; Sharopov, F.; Docea, A.O.; Karaca, A.C.; Sharifi-Rad, M.; Karincaoglu, D.K.; Gülseren, G.; Şenol, E.; Demircan, E.; et al. Diet, Lifestyle and Cardiovascular Diseases: Linking Pathophysiology to Cardioprotective Effects of Natural Bioactive Compounds. Int. J. Environ. Res. Public Health 2020, 17, 2326. [CrossRef]

15. Anuradha, C.V. Phytochemicals targeting genes relevant for type 2 diabetes. Can. J. Physiol. Pharmacol. 2013, 91, 397-411. [CrossRef] [PubMed]

16. Scarmeas, N.; Anastasiou, C.A.; Yannakoulia, M. Nutrition and prevention of cognitive impairment. Lancet Neurol. 2018, 17, 1006-1015. [CrossRef]

17. Grodzicki, W.; Dziendzikowska, K. The role of selected bioactive compounds in the prevention of alzheimer's disease. [El papel de los compuestos bioactivos seleccionados en la prevención de la enfermedad de Alzheimer]. Antioxidants 2020, 9, 229. [CrossRef] [PubMed]

18. Biesalski, H.K.; Dragsted, L.O.; Elmadfa, I.; Grossklaus, R.; Muller, M.; Schrenk, D.; Walter, P.; Weber, P. Bioactive compounds: Definition and assessment of activity. Nutrition 2009, 25, 1202-1205. [CrossRef] 
19. Slavin, J.L.; Lloyd, B. Health benefits of fruits and vegetables. Adv. Nutr. 2012, 3, 506-516. [CrossRef]

20. Kris-Etherton, P.M.; Hecker, K.D.; Bonanome, A.; Coval, S.M.; Binkoski, A.E.; Hilpert, K.F.; Griel, A.E.; Etherton, T.D. Bioactive compounds in foods: Their role in the prevention of cardiovascular disease and cancer. Am. J. Med. 2002, 113, 71-88. [CrossRef]

21. Sharif, M.K.; Khalid, R. Nutraceuticals: Myths Versus Realities. In Handbook of Food Bioengineering, Therapeutic Foods; Holban, A.M., Grumezescu, A.M., Eds.; Elsevier: Amsterdam, The Netherlands, 2018; pp. 3-21. [CrossRef]

22. Hernandez, D.F.; Cervantes, E.L.; Luna-Vital, D.A.; Mojica, L. Food-derived bioactive compounds with anti-aging potential for nutricosmetic and cosmeceutical products. Crit. Rev. Food Sci. Nutr. 2021, 61, 3740-3755. [CrossRef]

23. Marcillo-Parra, V.; Tupuna-Yerovi, D.S.; González, Z.; Ruales, J. Encapsulation of bioactive compounds from fruit and vegetable by-products for food application-A review. Trends Food Sci. Technol. 2021, 116, 11-23. [CrossRef]

24. Rodriguez-Morato, J.; Xicota, L.; Fito, M.; Farre, M.; Dierssen, M.; de la Torre, R. Potential role of olive oil phenolic compounds in the prevention of neurodegenerative diseases. Molecules 2015, 20, 4655-4680. [CrossRef]

25. Martin-Pelaez, S.; Covas, M.I.; Fito, M.; Kusar, A.; Pravst, I. Health effects of olive oil polyphenols: Recent advances and possibilities for the use of health claims. Mol. Nutr. Food Res. 2013, 57, 760-771. [CrossRef]

26. Venigalla, M.; Gyengesi, E.; Munch, G. Curcumin and Apigenin-Novel and promising therapeutics against chronic neuroinflammation in Alzheimer's disease. Neural Regen. Res. 2015, 10, 1181-1185. [CrossRef] [PubMed]

27. Camara, J.S.; Albuquerque, B.R.; Aguiar, J.; Correa, R.C.G.; Goncalves, J.L.; Granato, D.; Pereira, J.A.M.; Barros, L.; Ferreira, I. Food Bioactive Compounds and Emerging Techniques for Their Extraction: Polyphenols as a Case Study. Foods 2020, 10, 37. [CrossRef] [PubMed]

28. Kevers, C.; Falkowski, M.; Tabart, J.; Defraigne, J.O.; Dommes, J.; Pincemail, J. Evolution of antioxidant capacity during storage of selected fruits and vegetables. J. Agric. Food. Chem. 2007, 55, 8596-8603. [CrossRef] [PubMed]

29. Havsteen, B.H. The biochemistry and medical significance of the flavonoids. Pharmacol. Ther. 2002, 96, 67-202. [CrossRef]

30. Li, D.; Wang, P.; Luo, Y.; Zhao, M.; Chen, F. Health benefits of anthocyanins and molecular mechanisms: Update from recent decade. Crit. Rev. Food Sci. Nutr. 2017, 57, 1729-1741. [CrossRef]

31. Devore, E.E.; Kang, J.H.; Breteler, M.M.; Grodstein, F. Dietary intakes of berries and flavonoids in relation to cognitive decline. Ann. Neurol. 2012, 72, 135-143. [CrossRef]

32. Li, Y.; Ding, Y. Minireview: Therapeutic potential of myricetin in diabetes mellitus. Food Sci. Hum. Wellness 2012, 1, 19-25. [CrossRef]

33. Karasawa, M.M.G.; Mohan, C. Fruits as Prospective Reserves of bioactive Compounds: A Review. Nat Prod Bioprospect 2018, 8 , 335-346. [CrossRef]

34. Devi, K.P.; Shanmuganathan, B.; Manayi, A.; Nabavi, S.F.; Nabavi, S.M. Molecular and Therapeutic Targets of Genistein in Alzheimer's Disease. Mol. Neurobiol. 2017, 54, 7028-7041. [CrossRef]

35. Karas, M.; Jakubczyk, A.; Szymanowska, U.; Zlotek, U.; Zielinska, E. Digestion and bioavailability of bioactive phytochemicals. Int. J. Food Sci. Technol. 2017, 52, 291-305. [CrossRef]

36. Habanova, M.; Saraiva, J.A.; Haban, M.; Schwarzova, M.; Chlebo, P.; Predna, L.; Gazo, J.; Wyka, J. Intake of bilberries (Vaccinium myrtillus L.) reduced risk factors for cardiovascular disease by inducing favorable changes in lipoprotein profiles. Nutr. Res. 2016, 36, 1415-1422. [CrossRef] [PubMed]

37. Chao, S.C.; Chen, Y.J.; Huang, K.H.; Kuo, K.L.; Yang, T.H.; Huang, K.Y.; Wang, C.C.; Tang, C.H.; Yang, R.S.; Liu, S.H. Induction of sirtuin-1 signaling by resveratrol induces human chondrosarcoma cell apoptosis and exhibits antitumor activity. Sci. Rep. 2017, 7, 3180. [CrossRef] [PubMed]

38. Krinsky, N.I.; Johnson, E.J. Carotenoid actions and their relation to health and disease. Mol. Asp. Med. 2005, 26, 459-516. [CrossRef] [PubMed]

39. Davey, M.W.; Keulemans, J.; Swennen, R. Methods for the efficient quantification of fruit provitamin A contents. J. Chromatogr. A 2006, 1136, 176-184. [CrossRef]

40. Lakey-Beitia, J.; Doens, D.; Jagadeesh Kumar, D.; Murillo, E.; Fernandez, P.L.; Rao, K.S.; Durant-Archibold, A.A. Anti-amyloid aggregation activity of novel carotenoids: Implications for Alzheimer's drug discovery. Clin. Interv. Aging 2017, 12, 815-822. [CrossRef]

41. Hooper, L.; Cassidy, A. A review of the health care potential of bioactive compounds. J. Sci. Food Agric. 2006, 86, 1805-1813. [CrossRef]

42. Burcul, F.; Generalic Mekinic, I.; Radan, M.; Rollin, P.; Blazevic, I. Isothiocyanates: Cholinesterase inhibiting, antioxidant, and anti-inflammatory activity. J. Enzym. Inhib. Med. Chem. 2018, 33, 577-582. [CrossRef]

43. Marangoni, F.; Poli, A. Phytosterols and cardiovascular health. Pharmacol. Res. 2010, 61, 193-199. [CrossRef]

44. Verhaar, M.C.; Stroes, E.; Rabelink, T.J. Folates and cardiovascular disease. Arterioscler. Thromb. Vasc. Biol. 2002, 22 , 6-13. [CrossRef]

45. Saari, J.C.; Nawrot, M.; Kennedy, B.N.; Garwin, G.G.; Hurley, J.B.; Huang, J.; Possin, D.E.; Crabb, J.W. Visual cycle impairment in cellular retinaldehyde binding protein (CRALBP) knockout mice results in delayed dark adaptation. Neuron 2001, 29, 739-748. [CrossRef]

46. Stewart, L.M.V.; Weigel, N.L. Vitamin D and Prostate Cancer. In Experimental Biology and Medicine; Society for Experimental Biology and Medicine: New York, NY, USA, 2004; Volume 229, pp. 277-284. 
47. Norman, A.W. Minireview: Vitamin D receptor: New assignments for an already busy receptor. Endocrinology 2006, $147,5542-5548$. [CrossRef] [PubMed]

48. Boccardi, V.; Baroni, M.; Mangialasche, F.; Mecocci, P. Vitamin E family: Role in the pathogenesis and treatment of Alzheimer's disease. Alzheimers Dement. 2016, 2, 182-191. [CrossRef] [PubMed]

49. Vermeer, C.; Theuwissen, E. Vitamin K, osteoporosis and degenerative diseases of ageing. Menopause Int. 2011, 17, 19-23. [CrossRef] [PubMed]

50. Khattak, K.F.; Rahman, T.U. Analysis of vegetable's peels as a natural source of vitamins and minerals. Int. Food Res. J. 2017, 24, 292-297.

51. Tedesco, D.E.A.; Scarioni, S.; Tava, A.; Panseri, S.; Zuorro, A. Fruit and Vegetable Wholesale Market Waste: Safety and Nutritional Characterisation for Their Potential Re-Use in Livestock Nutrition. Sustainability 2021, 13, 9478. [CrossRef]

52. Villagran, M.; Ferreira, J.; Martorell, M.; Mardones, L. The Role of Vitamin C in Cancer Prevention and Therapy: A Literature Review. Antioxid 2021, 10, 1894. [CrossRef]

53. Piquereau, J.; Boitard, S.E.; Ventura-Clapier, R.; Mericskay, M. Metabolic Therapy of Heart Failure: Is There a Future for B Vitamins? Int. J. Mol. Sci. 2021, 23, 30. [CrossRef]

54. Dhingra, D.; Michael, M.; Rajput, H.; Patil, R.T. Dietary fibre in foods: A review. J. Food Sci. Technol. 2012, 49, 255-266. [CrossRef]

55. Devries, J.W. Dietary fiber: The influence of definition on analysis and regulation. J. AOAC Int. 2004, 87, 682-706. [CrossRef]

56. Fernandez-Garcia, E.; Carvajal-Lerida, I.; Perez-Galvez, A. In vitro bioaccessibility assessment as a prediction tool of nutritional efficiency. Nutr. Res. 2009, 29, 751-760. [CrossRef]

57. Manach, C.; Scalbert, A.; Morand, C.; Remesy, C.; Jimenez, L. Polyphenols: Food sources and bioavailability. Am. J. Clin. Nutr. 2004, 79, 727-747. [CrossRef] [PubMed]

58. Saura-Calixto, F.; Serrano, J.; Goni, I. Intake and bioaccessibility of total polyphenols in a whole diet. Food Chem. 2007, 101, 492-501. [CrossRef]

59. Fernandez-Garcia, E.; Carvajal-Lerida, I.; Jaren-Galan, M.; Garrido-Fernandez, J.; Perez-Galvez, A.; Hornero-Mendez, D. Carotenoids bioavailability from foods: From plant pigments to efficient biological activities. Food Res. Int. 2012, 46, 438450. [CrossRef]

60. Manach, C.; Williamson, G.; Morand, C.; Scalbert, A.; Remesy, C. Bioavailability and bioefficacy of polyphenols in humans. I. Review of 97 bioavailability studies. Am. J. Clin. Nutr. 2005, 81, 230S-242S. [CrossRef] [PubMed]

61. D'Archivio, M.; Filesi, C.; Vari, R.; Scazzocchio, B.; Masella, R. Bioavailability of the polyphenols: Status and controversies. Int. J. Mol. Sci. 2010, 11, 1321-1342. [CrossRef]

62. Lafay, S.; Gil-Izquierdo, A. Bioavailability of phenolic acids. Phytochem. Rev. 2007, 7, 301-311. [CrossRef]

63. Olthof, M.R.; Hollman, P.C.; Zock, P.L.; Katan, M.B. Consumption of high doses of chlorogenic acid, present in coffee, or of black tea increases plasma total homocysteine concentrations in humans. Am. J. Clin. Nutr. 2001, 73, 532-538. [CrossRef]

64. Falcone Ferreyra, M.L.; Rius, S.P.; Casati, P. Flavonoids: Biosynthesis, biological functions, and biotechnological applications. Front. Plant Sci. 2012, 3, 222. [CrossRef]

65. Ozdal, T.; Sela, D.A.; Xiao, J.; Boyacioglu, D.; Chen, F.; Capanoglu, E. The Reciprocal Interactions between Polyphenols and Gut Microbiota and Effects on Bioaccessibility. Nutrients 2016, 8, 78. [CrossRef]

66. Fang, J. Bioavailability of anthocyanins. Drug Metab. Rev. 2014, 46, 508-520. [CrossRef]

67. McClements, D.J.; Xiao, H. Excipient foods: Designing food matrices that improve the oral bioavailability of pharmaceuticals and nutraceuticals. Food Funct. 2014, 5, 1320-1333. [CrossRef] [PubMed]

68. Carmody, R.N.; Wrangham, R.W. The energetic significance of cooking. J. Hum. Evol. 2009, 57, 379-391. [CrossRef] [PubMed]

69. Palermo, M.; Pellegrini, N.; Fogliano, V. The effect of cooking on the phytochemical content of vegetables. J. Sci. Food Agric. 2014, 94, 1057-1070. [CrossRef] [PubMed]

70. Munoz-Esparza, N.C.; Costa-Catala, J.; Comas-Baste, O.; Toro-Funes, N.; Latorre-Moratalla, M.L.; Veciana-Nogues, M.T.; VidalCarou, M.C. Occurrence of Polyamines in Foods and the Influence of Cooking Processes. Foods 2021, 10, 1752. [CrossRef] [PubMed]

71. Rinaldi, M.; Santi, S.; Paciulli, M.; Ganino, T.; Pellegrini, N.; Visconti, A.; Vitaglione, P.; Barbanti, D.; Chiavaro, E. Comparison of physical, microstructural and antioxidative properties of pumpkin cubes cooked by conventional, vacuum cooking and sous vide methods. J. Sci. Food Agric. 2021, 101, 2534-2541. [CrossRef]

72. Gabrielle Alves de Carvalho, A.; Olmo-Garcia, L.; Rachel Antunes Gaspar, B.; Carrasco-Pancorbo, A.; Naciuk Castelo-Branco, V.; Guedes Torres, A. Evolution of the metabolic profile of virgin olive oil during deep-frying: Assessing the transfer of bioactive compounds to the fried food. Food Chem. 2022, 380, 132205. [CrossRef]

73. Ojeda-Linares, C.; Alvarez-Rios, G.D.; Figueredo-Urbina, C.J.; Islas, L.A.; Lappe-Oliveras, P.; Nabhan, G.P.; Torres-Garcia, I.; Vallejo, M.; Casas, A. Traditional Fermented Beverages of Mexico: A Biocultural Unseen Foodscape. Foods 2021, 10, 2390. [CrossRef]

74. Di Cagno, R.; Filannino, P.; Gobbetti, M. Fermented Foods: Fermented Vegetables and Other Products. In Encyclopedia of Food and Health; Caballero, B., Finglas, P.M., Toldrá, F., Eds.; Academic Press: Oxford, UK, 2016; pp. 668-674. [CrossRef]

75. Park, K.-Y.; Jeong, J.-K. Chapter 26-Kimchi (Korean Fermented Vegetables) as a Probiotic Food. In Probiotics, Prebiotics, and Synbiotics; Watson, R.R., Preedy, V.R., Eds.; Academic Press: Cambridge, MA, USA, 2016; pp. 391-408. [CrossRef] 
76. Ansorena, D.; Astiasarán, I. Fermented Foods: Composition and Health effects. In Encyclopedia of Food and Health; Caballero, B., Finglas, P.M., Toldrá, F., Eds.; Academic Press: Oxford, UK, 2016; pp. 649-655. [CrossRef]

77. Koli, R.; Erlund, I.; Jula, A.; Marniemi, J.; Mattila, P.; Alfthan, G. Bioavailability of Various Polyphenols from a Diet Containing Moderate Amounts of Berries. J. Agric. Food. Chem. 2010, 58, 3927-3932. [CrossRef]

78. Ribas-Agusti, A.; Martin-Belloso, O.; Soliva-Fortuny, R.; Elez-Martinez, P. Food processing strategies to enhance phenolic compounds bioaccessibility and bioavailability in plant-based foods. Crit. Rev. Food Sci. Nutr. 2018, 58, 2531-2548. [CrossRef]

79. Caballero, B. Encyclopedia of Human Nutrition; Elsevier: Amsterdam, The Netherlands, 2005.

80. Arfaoui, L. Dietary Plant Polyphenols: Effects of Food Processing on Their Content and Bioavailability. Molecules 2021, 26, 2959. [CrossRef]

81. Almeida, D.P.F.; Gião, M.S.; Pintado, M.; Gomes, M.H. Bioactive phytochemicals in apple cultivars from the Portuguese protected geographical indication "Maçã de Alcobaça:" Basis for market segmentation. Int. J. Food Prop. 2017, 20, 2206-2214. [CrossRef]

82. Loncaric, A.; Matanovic, K.; Ferrer, P.; Kovac, T.; Sarkanj, B.; Skendrovic Babojelic, M.; Lores, M. Peel of Traditional Apple Varieties as a Great Source of Bioactive Compounds: Extraction by Micro-Matrix Solid-Phase Dispersion. Foods 2020, 9, 80. [CrossRef] [PubMed]

83. Viroli, S.L.M.; Viroli, S.G.; Carvalho, N.P.; Bernardi, D.P.d.S.; Coelho, R.G. Production and characterization of acetic fermentation with different fruit peels. Res. Soc. Dev. 2021, 10, e84101421878. [CrossRef]

84. Singh, B.; Singh, J.P.; Kaur, A.; Singh, N. Bioactive compounds in banana and their associated health benefits-A review. Food Chem. 2016, 206, 1-11. [CrossRef] [PubMed]

85. Oyeyinka, B.O.; Afolayan, A.J. Comparative Evaluation of the Nutritive, Mineral, and Antinutritive Composition of Musa sinensis L. (Banana) and Musa paradisiaca L. (Plantain) Fruit Compartments. Plants 2019, 8, 598. [CrossRef] [PubMed]

86. Kesa, A.L.; Pop, C.R.; Mudura, E.; Salanta, L.C.; Pasqualone, A.; Darab, C.; Burja-Udrea, C.; Zhao, H.; Coldea, T.E. Strategies to Improve the Potential Functionality of Fruit-Based Fermented Beverages. Plants 2021, 10, 2263. [CrossRef]

87. Muhammad Suffi, N.S.; Mohamed, E.; Camalxaman, S.N.; Rambely, A.S.; Haron, N. The medicinal benefits, phytochemical constituents and antioxidant properties of banana blossom: A mini review. Healthscope Off. Res. Book Fac. Health Sci. UiTM 2021, $4,113-118$.

88. M'hiri, N.; Ioannou, I.; Ghoul, M.; Boudhrioua, N.M. Phytochemical characteristics of citrus peel and effect of conventional and nonconventional processing on phenolic compounds: A review. Food Rev. Int. 2017, 33, 587-619. [CrossRef]

89. Chavan, P.; Singh, A.K.; Kaur, G. Recent progress in the utilization of industrial waste and by-products of citrus fruits: A review. J. Food Process Eng. 2018, 41, e12895. [CrossRef]

90. Sir Elkhatim, K.A.; Elagib, R.A.A.; Hassan, A.B. Content of phenolic compounds and vitamin C and antioxidant activity in wasted parts of Sudanese citrus fruits. Food Sci. Nutr. 2018, 6, 1214-1219. [CrossRef]

91. Perestrelo, R.; Lu, Y.; Santos, S.A.O.; Silvestre, A.J.D.; Neto, C.P.; Câmara, J.S.; Rocha, S.M. Phenolic profile of Sercial and Tinta Negra Vitis vinifera L. grape skins by HPLC-DAD-ESI-MSn. Food Chem. 2012, 135, 94-104. [CrossRef]

92. Iora, S.R.F.; Maciel, G.M.; Zielinski, A.A.F.; da Silva, M.V.; Pontes, P.V.D.; Haminiuk, C.W.I.; Granato, D. Evaluation of the bioactive compounds and the antioxidant capacity of grape pomace. Int. J. Food Sci. Technol. 2015, 50, 62-69. [CrossRef]

93. Beres, C.; Costa, G.N.S.; Cabezudo, I.; da Silva-James, N.K.; Teles, A.S.C.; Cruz, A.P.G.; Mellinger-Silva, C.; Tonon, R.V.; Cabral, L.M.C.; Freitas, S.P. Towards integral utilization of grape pomace from winemaking process: A review. Waste Manag. 2017, 68, 581-594. [CrossRef] [PubMed]

94. Pintac, D.; Majkic, T.; Torovic, L.; Orcic, D.; Beara, I.; Simin, N.; Mimica-Dukic, N.; Lesjak, M. Solvent selection for efficient extraction of bioactive compounds from grape pomace. Ind. Crops Prod. 2018, 111, 379-390. [CrossRef]

95. Peixoto, C.M.; Dias, M.I.; Alves, M.J.; Calhelha, R.C.; Barros, L.; Pinho, S.P.; Ferreira, I. Grape pomace as a source of phenolic compounds and diverse bioactive properties. Food Chem. 2018, 253, 132-138. [CrossRef] [PubMed]

96. Antonic, B.; Jancikova, S.; Dordevic, D.; Tremlova, B. Grape Pomace Valorization: A Systematic Review and Meta-Analysis. Foods 2020, 9, 1627. [CrossRef]

97. Monteiro, G.C.; Minatel, I.O.; Pimentel, A.; Gomez-Gomez, H.A.; de Camargo, J.P.C.; Diamante, M.S.; Basilio, L.S.P.; Tecchio, M.A.; Lima, G.P.P. Bioactive compounds and antioxidant capacity of grape pomace flours. Lwt-Food Sci. Technol. 2021, 135, 110053. [CrossRef]

98. Perra, M.; Lozano-Sanchez, J.; Leyva-Jimenez, F.J.; Segura-Carretero, A.; Pedraz, J.L.; Bacchetta, G.; Muntoni, A.; De Gioannis, G.; Manca, M.L.; Manconi, M. Extraction of the antioxidant phytocomplex from wine-making by-products and sustainable loading in phospholipid vesicles specifically tailored for skin protection. Biomed. Pharmacother. 2021, 142, 111959. [CrossRef]

99. Dias, M.; Caleja, C.; Pereira, C.; Calhelha, R.C.; Kostic, M.; Sokovic, M.; Tavares, D.; Baraldi, I.J.; Barros, L.; Ferreira, I. Chemical composition and bioactive properties of byproducts from two different kiwi varieties. Food Res. Int. 2020, 127, 108753. [CrossRef]

100. Satpal, D.; Kaur, J.; Bhadariya, V.; Sharma, K. Actinidia deliciosa (Kiwi fruit): A comprehensive review on the nutritional composition, health benefits, traditional utilization, and commercialization. J. Food Process. Preserv. 2021, 45, e15588. [CrossRef]

101. Chamorro, F.; Carpena, M.; Fraga-Corral, M.; Echave, J.; Riaz Rajoka, M.S.; Barba, F.J.; Cao, H.; Xiao, J.; Prieto, M.A.; SimalGandara, J. Valorization of kiwi agricultural waste and industry by-products by recovering bioactive compounds and applications as food additives: A circular economy model. Food Chem. 2022, 370, 131315. [CrossRef] [PubMed]

102. Jahurul, M.H.; Zaidul, I.S.; Ghafoor, K.; Al-Juhaimi, F.Y.; Nyam, K.L.; Norulaini, N.A.; Sahena, F.; Mohd Omar, A.K. Mango (Mangifera indica L.) by-products and their valuable components: A review. Food Chem. 2015, 183, 173-180. [CrossRef] [PubMed] 
103. Mugwagwa, L.R.; Chimphango, A.F.A. Box-Behnken design based multi-objective optimisation of sequential extraction of pectin and anthocyanins from mango peels. Carbohydr. Polym. 2019, 219, 29-38. [CrossRef]

104. Hazarudin, N.H.; Razali, N.F. A Review on Extraction of Antioxidants from Mangifera indica L. (Mango) Peel and Seed with Highest Extraction Yield. Prog. Eng. Appl. Technol. 2021, 2, 699-710.

105. Lebaka, V.R.; Wee, Y.J.; Ye, W.; Korivi, M. Nutritional Composition and Bioactive Compounds in Three Different Parts of Mango Fruit. Int. J. Environ. Res. Public Health 2021, 18, 741. [CrossRef]

106. Quintana, S.E.; Salas, S.; Garcia-Zapateiro, L.A. Bioactive compounds of mango (Mangifera indica): A review of extraction technologies and chemical constituents. J. Sci. Food Agric. 2021, 101, 6186-6192. [CrossRef] [PubMed]

107. Vigano, J.; Brumer, I.Z.; Braga, P.A.D.; da Silva, J.K.; Marostica, M.R.; Reyes, F.G.R.; Martinez, J. Pressurized liquids extraction as an alternative process to readily obtain bioactive compounds from passion fruit rinds. Food Bioprod. Process. 2016, 100, 382-390. [CrossRef]

108. Coelho, E.M.; Gomes, R.G.; Machado, B.A.S.; Oliveira, R.S.; Lima, M.d.S.; de Azêvedo, L.C.; Guez, M.A.U. Passion fruit peel flour-Technological properties and application in food products. Food Hydrocoll. 2017, 62, 158-164. [CrossRef]

109. Morais, D.R.; Rotta, E.M.; Sargi, S.C.; Bonafe, E.G.; Suzuki, R.M.; Souza, N.E.; Matsushita, M.; Visentainer, J.V. Proximate Composition, Mineral Contents and Fatty Acid Composition of the Different Parts and Dried Peels of Tropical Fruits Cultivated in Brazil. J. Braz. Chem. Soc. 2017, 28, 308-318. [CrossRef]

110. Mandal, G.; Thokchom, R. Production Preference and Importance of Passion Fruit (Passiflora Edulis): A Review. J. Agric. Eng. Food Technol. 2017, 4, 27-30.

111. Coelho, E.M.; de Azevedo, L.C.; Viana, A.C.; Ramos, I.G.; Gomes, R.G.; Lima, M.D.S.; Umsza-Guez, M.A. Physico-chemical properties, rheology and degree of esterification of passion fruit (Passiflora edulis f. flavicarpa) peel flour. J. Sci. Food Agric. 2018, 98, 166-173. [CrossRef] [PubMed]

112. Moia, T.A.; Pimentel, T.C.; Barão, C.E.; Feihrmann, A.C.; Favareto, R.; Reis, A.V.; Cardozo-Filho, L. Bioactive compounds and pectin from residues of the passion fruit processing: Extraction using Green Technology and Characterization. Chem. Eng. Trans. 2019, 75, 157-162. [CrossRef]

113. Dominguez-Rodriguez, G.; Garcia, M.C.; Plaza, M.; Marina, M.L. Revalorization of Passiflora species peels as a sustainable source of antioxidant phenolic compounds. Sci. Total Environ. 2019, 696, 134030. [CrossRef]

114. Reis, L.C.R.D.; Facco, E.M.P.; Salvador, M.; Flôres, S.H.; Rios, A.d.O. Characterization of Orange Passion Fruit Peel Flour and Its Use as an Ingredient in Bakery Products. J. Culin. Sci. Technol. 2018, 18, 214-230. [CrossRef]

115. Pereira, D.T.V.; Zabot, G.L.; Reyes, F.G.; Iglesias, A.H.; Martinez, J. Integration of pressurized liquids and ultrasound in the extraction of bioactive compounds from passion fruit rinds: Impact on phenolic yield, extraction kinetics and technical-economic evaluation. Innov. Food Sci. Emerg. Technol. 2021, 67. [CrossRef]

116. Li, X.; Wang, T.; Zhou, B.; Gao, W.; Cao, J.; Huang, L. Chemical composition and antioxidant and anti-inflammatory potential of peels and flesh from 10 different pear varieties (Pyrus spp.). Food Chem. 2014, 152, 531-538. [CrossRef]

117. Hong, S.Y.; Lansky, E.; Kang, S.S.; Yang, M. A review of pears (Pyrus spp.), ancient functional food for modern times. BMC Complement. Med. 2021, 21, 219. [CrossRef]

118. Loizzo, M.R.; Pacetti, D.; Lucci, P.; Nunez, O.; Menichini, F.; Frega, N.G.; Tundis, R. Prunus persica var. platycarpa (Tabacchiera Peach): Bioactive Compounds and Antioxidant Activity of Pulp, Peel and Seed Ethanolic Extracts. Plant Foods Hum. Nutr. 2015, 70, 331-337. [CrossRef]

119. Machado, M.I.R.; Machado, A.R.; Zambiazi, R.C. Pêssego: Características Físico-Químicas e Conteúdo de Compostos Bioativos. Res. Soc. Dev. 2020, 9, e216973103. [CrossRef]

120. Kan, J.A.; Chen, C.C.; Huo, T.B.; Xie, W.J.; Hui, Y.Y.; Liu, J.; Jin, C.H. Polyphenolic-enriched peach peels extract regulates lipid metabolism and improves the gut microbiota composition in high fat diet-fed mice. J. Funct. Foods 2020, 72, 104082. [CrossRef]

121. Valdes Garcia, A.; Domingo Martinez, M.I.; Ponce Landete, M.; Prats Moya, M.S.; Beltran Sanahuja, A. Potential of Industrial Pineapple (Ananas comosus (L.) Merrill) By-Products as Aromatic and Antioxidant Sources. Antioxid 2021, 10, 1767. [CrossRef] [PubMed]

122. Hikal, W.M.; Mahmoud, A.A.; Said-Al Ahl, H.A.H.; Bratovcic, A.; Tkachenko, K.G.; Kačániová, M.; Rodriguez, R.M. Pineapple (Ananas comosus L. Merr.), Waste Streams, Characterisation and Valorisation: An Overview. Open J. Ecol. 2021, 11, 610-634. [CrossRef]

123. Vieira, I.M.M.; Santos, B.L.P.; Santos, C.V.M.; Ruzene, D.S.; Silva, D.P. Valorization of Pineapple Waste: A Review on How the Fruit's Potential Can Reduce Residue Generation. BioEnergy Res. 2021. [CrossRef]

124. Medina-Meza, I.G.; Barbosa-Canovas, G.V. Assisted extraction of bioactive compounds from plum and grape peels by ultrasonics and pulsed electric fields. J. Food Eng. 2015, 166, 268-275. [CrossRef]

125. Sarmento, J.D.A.; de Morais, P.L.D.; de Souza, F.I.; da Costa, L.R.; Melo, N.J.D. Bioactive compounds and antioxidant activity of Ximenia americana coming from different collection sites. Arch. Latinoam. Nutr. 2015, 65, 263-270.

126. Hernandez-Ruiz, K.L.; Ruiz-Cruz, S.; Cira-Chavez, L.A.; Gassos-Ortega, L.E.; Ornelas-Paz, J.J.; Del-Toro-Sanchez, C.L.; MarquezRios, E.; Lopez-Mata, M.A.; Rodriguez-Felix, F. Evaluation of Antioxidant Capacity, Protective Effect on Human Erythrocytes and Phenolic Compound Identification in Two Varieties of Plum Fruit (Spondias spp.) by UPLC-MS. Molecules 2018, $23,3200$. [CrossRef] 
127. Rodriguez-Miranda, J.; Hernandez-Santos, B.; Herman-Lara, E.; Vivar-Vera, M.A.; Carmona-Garcia, R.; Gomez-Aldapa, C.A.; Martinez-Sanchez, C.E. Physicochemical and functional properties of whole and defatted meals from Mexican (Cucurbita pepo) pumpkin seeds. Int. J. Food Sci. Technol. 2012, 47, 2297-2303. [CrossRef]

128. Montesano, D.; Blasi, F.; Simonetti, M.S.; Santini, A.; Cossignani, L. Chemical and Nutritional Characterization of Seed Oil from Cucurbita maxima L. (var. Berrettina) Pumpkin. Foods 2018, 7, 30. [CrossRef]

129. Kulczynski, B.; Gramza-Michalowska, A. The Profile of Secondary Metabolites and Other Bioactive Compounds in Cucurbita pepo L. and Cucurbita moschata Pumpkin Cultivars. Molecules 2019, 24, 2945. [CrossRef]

130. Ahmad, G.; Khan, A.A. Pumpkin: Horticultural Importance and Its Roles in Various Forms; a Review. Int. J. Hortic. Agric. 2019, 4, 1-6. [CrossRef]

131. WHO, World Health Organization. Diet, Nutrition and the Prevention of Chronic Diseases; WHO: Geneva, Switzerland, 2003.

132. Ferreira, D.F.; Barin, J.S.; Binello, A.; Veselou, V.V.; Cravotto, G. Highly efficient pumpkin-seed extraction with the simultaneous recovery of lipophilic and hydrophilic compounds. Food Bioprod. Process. 2019, 117, 224-230. [CrossRef]

133. Syed, Q.A. Nutritional and Therapeutic Importance of the Pumpkin Seeds. Biomed. J. Sci. Tech. Res. 2019, 21. [CrossRef]

134. Koltsov, V.A.; Danilin, S.I. Chemical composition of a seed kernel of a pumpkin produced in the Central Chernozem region of Russia. IOP Conf. Ser. Earth Environ. Sci. 2021, 845, 012004. [CrossRef]

135. Wani, A.A.; Sogi, D.S.; Singh, P.; Shivhare, U.S. Characterization and Functional Properties of Watermelon (Citrullus lanatus) Seed Protein Isolates and Salt Assisted Protein Concentrates. Food Sci. Biotechnol. 2011, 20, 877-887. [CrossRef]

136. Rezig, L.; Chouaibi, M.; Meddeb, W.; Msaada, K.; Hamdi, S. Chemical composition and bioactive compounds of Cucurbitaceae seeds: Potential sources for new trends of plant oils. Process Saf. Environ. Prot. 2019, 127, 73-81. [CrossRef]

137. Enemor, V.H.A.; Oguazu, C.E.; Odiakosa, A.U.; Okafor, S.C. Evaluation of the Medicinal Properties and Possible Nutrient Composition of Citrullus lanatus (Watermelon) Seeds. Res. J. Med. Plants 2019, 13, 129-135. [CrossRef]

138. Zia, S.; Khan, M.R.; Shabbir, M.A.; Aadil, R.M. An update on functional, nutraceutical and industrial applications of watermelon by-products: A comprehensive review. Trends Food Sci. Technol. 2021, 114, 275-291. [CrossRef]

139. Sorokina, M.; McCaffrey, K.S.; Deaton, E.E.; Ma, G.; Ordovas, J.M.; Perkins-Veazie, P.M.; Steinbeck, C.; Levi, A.; Parnell, L.D. A Catalog of Natural Products Occurring in Watermelon-Citrullus lanatus. Front. Nutr. 2021, 8, 729822. [CrossRef]

140. Ferreira, S.S.; Passos, C.P.; Cardoso, S.M.; Wessel, D.F.; Coimbra, M.A. Microwave assisted dehydration of broccoli by-products and simultaneous extraction of bioactive compounds. Food Chem. 2018, 246, 386-393. [CrossRef]

141. Petkowicz, C.L.O.; Williams, P.A. Pectins from food waste: Characterization and functional properties of a pectin extracted from broccoli stalk. Food Hydrocoll. 2020, 107, 105930. [CrossRef]

142. Xu, Y.Y.; Xiao, Y.D.; Lagnika, C.; Song, J.F.; Li, D.J.; Liu, C.Q.; Jiang, N.; Zhang, M.; Duan, X. A comparative study of drying methods on physical characteristics, nutritional properties and antioxidant capacity of broccoli. Dry. Technol. 2020, 38, 1378-1388. [CrossRef]

143. Vanlalneihi, B.; Saha, P.; Kalia, P.; Jaiswal, S.; Kundu, A.; Saha, N.D.; Sirowa, S.S.; Singh, N. Chemometric approach based characterization and selection of mid-early cauliflower for bioactive compounds and antioxidant activity. J. Food Sci. Technol. 2020, 57, 293-300. [CrossRef] [PubMed]

144. Drabinska, N.; Jez, M.; Nogueira, M. Variation in the Accumulation of Phytochemicals and Their Bioactive Properties among the Aerial Parts of Cauliflower. Antioxid 2021, 10, 1597. [CrossRef] [PubMed]

145. Mythili, S.; Rajeswari, N.; Bosco, S.J.D.; Rajalechumi, A.K.A. Impact of blanching treatments on the chemical composition, total dietary fiber, physicochemical, functional, and structural properties of underutilized cauliflower leaves (Brassica oleracea var. botrytis). J. Food Process. Preserv. 2021, 45, e15910. [CrossRef]

146. Zaheer, K.; Akhtar, M.H. Potato Production, Usage, and Nutrition-A Review. Crit. Rev. Food Sci. Nutr. 2016, 56, 711-721. [CrossRef]

147. Akyol, H.; Riciputi, Y.; Capanoglu, E.; Caboni, M.F.; Verardo, V. Phenolic Compounds in the Potato and Its Byproducts: An Overview. Int. J. Mol. Sci. 2016, 17, 835. [CrossRef] [PubMed]

148. Pathak, P.D.; Mandavgane, S.A.; Puranik, N.M.; Jambhulkar, S.J.; Kulkarni, B.D. Valorization of potato peel: A biorefinery approach. Crit. Rev. Biotechnol. 2018, 38, 218-230. [CrossRef]

149. Calcio Gaudino, E.; Colletti, A.; Grillo, G.; Tabasso, S.; Cravotto, G. Emerging Processing Technologies for the Recovery of Valuable Bioactive Compounds from Potato Peels. Foods 2020, 9, 1598. [CrossRef]

150. Sampaio, S.L.; Petropoulos, S.A.; Dias, M.I.; Pereira, C.; Calhelha, R.C.; Fernandes, A.; Leme, C.M.M.; Alexopoulos, A.; SantosBuelga, C.; Ferreira, I.; et al. Phenolic composition and cell-based biological activities of ten coloured potato peels (Solanum tuberosum L.). Food Chem. 2021, 363, 130360. [CrossRef]

151. Sharma, M.; Bhat, R. Extraction of Carotenoids from Pumpkin Peel and Pulp: Comparison between Innovative Green Extraction Technologies (Ultrasonic and Microwave-Assisted Extractions Using Corn Oil). Foods 2021, 10, 787. [CrossRef]

152. Lalnunthari, C.; Devi, L.M.; Badwaik, L.S. Extraction of protein and pectin from pumpkin industry by-products and their utilization for developing edible film. J. Food Sci. Technol. 2020, 57, 1807-1816. [CrossRef] [PubMed]

153. Pereira, A.; Maraschin, M. Banana (Musa spp.) from peel to pulp: Ethnopharmacology, source of bioactive compounds and its relevance for human health. J. Ethnopharmacol. 2015, 160, 149-163. [CrossRef] [PubMed] 
154. Borges, C.V.; Amorim, E.P.; Leonel, M.; Gomez, H.A.G.; dos Santos, T.P.R.; Ledo, C.A.D.; Belin, M.A.F.; de Almeida, S.L.; Minatel, I.O.; Lima, G.P.P. Post-harvest physicochemical profile and bioactive compounds of 19 bananas and plantains genotypes. Bragantia 2019, 78, 284-296. [CrossRef]

155. Mondal, A.; Banerjee, S.; Bose, S.; Das, P.P.; Sandberg, E.N.; Atanasov, A.G.; Bishayee, A. Cancer Preventive and Therapeutic Potential of Banana and Its Bioactive Constituents: A Systematic, Comprehensive, and Mechanistic Review. Front. Oncol. 2021, 11, 697143. [CrossRef]

156. Ling, S.S.; Chang, S.K.; Sia, W.C.; Yim, H.S. Antioxidant effcacy of unripe banana (Musa acuminata Colla) peel extracts in sunflower oil during accelerated storage. Acta Sci. Pol. Technol. Aliment. 2015, 14, 343-356. [CrossRef]

157. Mostafa, H.S. Banana plant as a source of valuable antimicrobial compounds and its current applications in the food sector. J. Food Sci. 2021, 86, 3778-3797. [CrossRef]

158. Toh, P.Y.; Leong, F.S.; Chang, S.K.; Khoo, H.E.; Yim, H.S. Optimization of extraction parameters on the antioxidant properties of banana waste. Acta Sci. Pol. Technol. Aliment. 2016, 15, 65-78. [CrossRef]

159. Borges, C.V.; Maraschin, M.; Coelho, D.S.; Leonel, M.; Gomez, H.A.G.; Belin, M.A.F.; Diamante, M.S.; Amorim, E.P.; Gianeti, T.; Castro, G.R.; et al. Nutritional value and antioxidant compounds during the ripening and after domestic cooking of bananas and plantains. Food Res. Int. 2020, 132, 109061. [CrossRef]

160. Kandasamy, S.; Aradhya, S.M. Polyphenolic profile and antioxidant properties of rhizome of commercial banana cultivars grown in India. Food Biosci. 2014, 8, 22-32. [CrossRef]

161. Chiang, S.H.; Yang, K.M.; Lai, Y.C.; Chen, C.W. Evaluation of the in vitro biological activities of Banana flower and bract extracts and their bioactive compounds. Int. J. Food Prop. 2021, 24, 1-16. [CrossRef]

162. Ramu, R.; Shirahatti, P.S.; Zameer, F.; Ranganatha, L.V.; Nagendra Prasad, M.N. Inhibitory effect of banana (Musa sp. var Nanjangud rasa bale) flower extract and its constituents Umbelliferone and Lupeol on $\alpha$-glucosidase, aldose reductase and glycation at multiple stages. S. Afr. J. Bot. 2014, 95, 54-63. [CrossRef]

163. Sheng, Z.; Dai, H.; Pan, S.; Ai, B.; Zheng, L.; Zheng, X.; Prinyawiwatkul, W.; Xu, Z. Phytosterols in banana (Musa spp.) flower inhibit $\alpha$-glucosidase and $\alpha$-amylase hydrolysations and glycation reaction. Int. J. Food Sci. Tech. 2017, 52, 171-179. [CrossRef]

164. Saini, R.K.; Ranjit, A.; Sharma, K.; Prasad, P.; Shang, X.; Gowda, K.G.M.; Keum, Y.S. Bioactive Compounds of Citrus Fruits: A Review of Composition and Health Benefits of Carotenoids, Flavonoids, Limonoids, and Terpenes. Antioxid 2022, 11, 239. [CrossRef]

165. Jeong, D.; Park, H.; Jang, B.K.; Ju, Y.; Shin, M.H.; Oh, E.J.; Lee, E.J.; Kim, S.R. Recent advances in the biological valorization of citrus peel waste into fuels and chemicals. Bioresour. Technol. 2021, 323, 124603. [CrossRef] [PubMed]

166. Yadav, V.; Sarker, A.; Yadav, A.; Miftah, A.O.; Bilal, M.; Iqbal, H.M.N. Integrated biorefinery approach to valorize citrus waste: A sustainable solution for resource recovery and environmental management. Chemosphere 2022, 293, 133459. [CrossRef] [PubMed]

167. Yaqoob, M.; Aggarwal, P.; Babbar, N. Extraction and screening of kinnow (Citrus reticulata L.) peel phytochemicals, grown in Punjab, India. Biomass Convers. Biorefin. 2022. [CrossRef]

168. Tsitsagi, M.; Ebralidze, K.; Chkhaidze, M.; Rubashvili, I.; Tsitsishvili, V. Sequential extraction of bioactive compounds from tangerine (Citrus Unshiu) peel. Ann. Agrar. Sci. 2018, 16, 236-241. [CrossRef]

169. Satari, B.; Karimi, K. Citrus processing wastes: Environmental impacts, recent advances, and future perspectives in total valorization. Resour. Conserv. Recycl. 2018, 129, 153-167. [CrossRef]

170. Figueira, J.A.; Porto-Figueira, P.; Pereira, J.A.M.; Camara, J.S. Tangerines Cultivated on Madeira Island-A High Throughput Natural Source of Bioactive Compounds. Foods 2020, 9, 1470. [CrossRef]

171. Makris, D.P.; Kallithraka, S.; Kefalas, P. Flavonols in grapes, grape products and wines: Burden, profile and influential parameters. J. Food Compos. Anal. 2006, 19, 396-404. [CrossRef]

172. de Andrade, R.B.; Machado, B.A.S.; Barreto, G.A.; Nascimento, R.Q.; Correa, L.C.; Leal, I.L.; Tavares, P.; Ferreira, E.S.; UmszaGuez, M.A. Syrah Grape Skin Residues Has Potential as Source of Antioxidant and Anti-Microbial Bioactive Compounds. Biology 2021, 10, 1262. [CrossRef] [PubMed]

173. Alim, A.; Li, T.; Nisar, T.; Ren, D.; Zhai, X.; Pang, Y.; Yang, X. Antioxidant, antimicrobial, and antiproliferative activity-based comparative study of peel and flesh polyphenols from Actinidia chinensis. Food Nutr. Res. 2019, 63, 1577. [CrossRef] [PubMed]

174. de Toledo, N.; de Camargo, A.; Ramos, P.; Button, D.; Granato, D.; Canniatti-Brazaca, S. Potentials and Pitfalls on the Use of Passion Fruit By-Products in Drinkable Yogurt: Physicochemical, Technological, Microbiological, and Sensory Aspects. Beverages 2018, 4, 47. [CrossRef]

175. Marinelli, V.; Lucera, A.; Incoronato, A.L.; Morcavallo, L.; Del Nobile, M.A.; Conte, A. Strategies for fortified sustainable food: The case of watermelon-based candy. J. Food Sci. Technol. 2021, 58, 894-901. [CrossRef] [PubMed]

176. Tarazona-Diaz, M.P.; Viegas, J.; Moldao-Martins, M.; Aguayo, E. Bioactive compounds from flesh and by-product of fresh-cut watermelon cultivars. J. Sci. Food Agric. 2011, 91, 805-812. [CrossRef] [PubMed]

177. Fadimu, G.J.; Ghafoor, K.; Babiker, E.E.; Al-Juhaimi, F.; Abdulraheem, R.A.; Adenekan, M.K. Ultrasound-assisted process for optimal recovery of phenolic compounds from watermelon (Citrullus lanatus) seed and peel. J. Food Meas. Charact. 2020, 14, 1784-1793. [CrossRef]

178. Bayat Mokhtari, R.; Baluch, N.; Homayouni, T.S.; Morgatskaya, E.; Kumar, S.; Kazemi, P.; Yeger, H. The role of Sulforaphane in cancer chemoprevention and health benefits: A mini-review. J. Cell Commun. Signal 2018, 12, 91-101. [CrossRef] 
179. Martinez-Fernandez, J.S.; Gu, X.Y.; Chen, S.L. Techno-economic assessment of bioactive compound recovery from potato peels with sequential hydrothermal extraction. J. Clean. Prod. 2021, 282, 124356. [CrossRef]

180. Rodriguez-Martinez, B.; Gullon, B.; Yanez, R. Identification and Recovery of Valuable Bioactive Compounds from Potato Peels: A Comprehensive Review. Antioxid 2021, 10, 1630. [CrossRef]

181. Friedman, M.; Huang, V.; Quiambao, Q.; Noritake, S.; Liu, J.; Kwon, O.; Chintalapati, S.; Young, J.; Levin, C.E.; Tam, C.; et al. Potato Peels and Their Bioactive Glycoalkaloids and Phenolic Compounds Inhibit the Growth of Pathogenic Trichomonads. J. Agric. Food Chem. 2018, 66, 7942-7947. [CrossRef]

182. Yesodharan, K.; Sujana, K.A. Ethnomedicinal knowledge among Malamalasar tribe of Parambikulam wildlife sanctuary, Kerala. Indian J. Tradit. Knowl. 2007, 6, 481-485.

183. Monica, K.; Agarwal, S.; Sanjay, P. Characterization of a chromaffin derivative isolated from the seeds of Ensete superbum Cheesm. Phcog. Mag. 2008, 4, 114-117.

184. Sasipriya, G.; Maria, C.L.; Siddhuraju, P. Influence of pressure cooking on antioxidant activity of wild (Ensete superbum) and commercial banana (Musa paradisiaca var. Monthan) unripe fruit and flower. J. Food Sci. Technol. 2014, 51, 2517-2525. [CrossRef] [PubMed]

185. Lawson, N. Cook, Eat, Repeat: Ingredients, Recipes and Stories.; ECCO: New York, NY, USA, 2021.

186. Gonzalez-Montelongo, R.; Lobo, M.G.; Gonzalez, M. Antioxidant activity in banana peel extracts: Testing extraction conditions and related bioactive compounds. Food Chem. 2010, 119, 1030-1039. [CrossRef]

187. Newgent, J. The Big Green Cookbook: Hundreds of Planet-Pleasing Recipes and Tips for a Luscious, Low-Carbon Lifestyle; Houghton Mifflin Harcourtv: San Francisco, SF, USA, 2009.

188. Sivakumaran, S.; Huffman, L.; Sivakumaran, S.; Drummond, L. The nutritional composition of Zespri(R) SunGold Kiwifruit and Zespri(R) Sweet Green Kiwifruit. Food Chem. 2018, 238, 195-202. [CrossRef]

189. Katz, S. Sandor Katz's Fermentation Journeys; Chelsea Green Publishing: Hartford, VT, USA, 2021.

190. Nakov, G.; Brandolini, A.; Hidalgo, A.; Ivanova, N.; Jukic, M.; Komlenic, D.K.; Lukinac, J. Influence of apple peel powder addition on the physico-chemical characteristics and nutritional quality of bread wheat cookies. Food Sci. Technol. Int. 2020, 26, 574-582 [CrossRef]

191. Teixeira, F.; Santos, B.A.D.; Nunes, G.; Soares, J.M.; Amaral, L.A.D.; Souza, G.H.O.; Resende, J.T.V.; Menegassi, B.; Rafacho, B.P.M.; Schwarz, K.; et al. Addition of Orange Peel in Orange Jam: Evaluation of Sensory, Physicochemical, and Nutritional Characteristics. Molecules 2020, 25, 1670. [CrossRef]

192. Ning, X.; Wu, J.; Luo, Z.; Chen, Y.; Mo, Z.; Luo, R.; Bai, C.; Du, W.; Wang, L. Cookies fortified with purple passion fruit epicarp flour: Impact on physical properties, nutrition, in vitro starch digestibility, and antioxidant activity. Cereal Chem. 2020, 98, 328-336. [CrossRef]

193. Zaki, N.A.M.; Rahman, N.A.; Zamanhuri, N.A.; Hashib, S.A. Ascorbic acid content and proteolytic enzyme activity of microwavedried pineapple stem and core. Chem. Eng. Trans. 2017, 56, 1369-1374. [CrossRef]

194. Shiau, S.Y.; Wu, M.Y.; Liu, Y.L. The effect of pineapple core fiber on dough rheology and the quality of mantou. J. Food Drug Anal. 2015, 23, 493-500. [CrossRef]

195. Hafid, H.; Patriani, P.; Sepriadi, S.; Ananda, S.H. Organoleptic properties of pineapple peel juice marinated beef (Ananas comosus L. Merr). E3S Web Conf. 2021, 332, 03005. [CrossRef]

196. Brigagao, T.C.S.; Fortes, R.R.; Lourenco, C.O.; Carvalho, E.E.N.; Cirillo, M.A.; Nachtigall, A.M.; Boas, B.M.V. Optimization of gluten-free muffins made with pineapple peel, banana peel, and pumpkin seed flours. J. Food Process. Preserv. 2021, 45 , e16037. [CrossRef]

197. Mohammadi-Moghaddam, T.; Firoozzare, A. Investigating the effect of sensory properties of black plum peel marmalade on consumers acceptance by Discriminant Analysis. Food Chem. X 2021, 11, 100126. [CrossRef] [PubMed]

198. Moghaddam, T.N.; Elhamirad, A.H.; Asl, M.R.S.; Noghabi, M.S. Pulsed electric field-assisted extraction of phenolic antioxidants from tropical almond red leaves. Chem. Pap. 2020, 74, 3957-3961. [CrossRef]

199. Durmaz, A.; Yuksel, F. Deep fried wheat chips added with potato peel flour-Effect on quality parameters. Qual. Assur. Saf. Crops Foods 2021, 13, 115-124. [CrossRef]

200. Ben Jeddou, K.; Bouaziz, F.; Zouari-Ellouzi, S.; Chaari, F.; Ellouz-Chaabouni, S.; Ellouz-Ghorbel, R.; Nouri-Ellouz, O. Improvement of texture and sensory properties of cakes by addition of potato peel powder with high level of dietary fiber and protein. Food Chem. 2017, 217, 668-677. [CrossRef] [PubMed]

201. Jacinto, G.; Stieven, A.; Maciel, M.J.; Souza, C.F.V.d. Effect of potato peel, pumpkin seed, and quinoa flours on sensory and chemical characteristics of gluten-free breads. Braz. J. Food Technol. 2020, 23, 2020. [CrossRef]

202. Kl, N.; Lau, M.; Cp, T. Fibre from Pumpkin (C. pepo L.) Seeds and Rinds: Application as Bakery Product Ingredients Fibre from Pumpkin (Cucurbita pepo L.) Seeds and Rinds: Physico-chemical Properties, Antioxidant Capacity and Application as Bakery Product Ingredients. Mal. J. Nutr. 2013, 19, 99-109.

203. Zabala, V.B.; Goles, C.E. Watermelon Rind-Ponkan Marmalade: A Physico-chemical Analysis. J. Phys. Conf. Ser. 2021, $1835,012114$. [CrossRef] 
204. Adegunwa, M.O.; Oloyede, I.O.; Adebanjo, L.A.; Alamu, E.O. Quality attribute of plantain (Musa paradisiaca) sponge-cake supplemented with watermelon (Citrullus lanatus) rind flour. Cogent Food Agric. 2019, 5, 1631582. [CrossRef]

205. Raja, M.P.; Praveen Raja, M.; Karthiayani, A.; Selvan, P.; Nithyalakshmi, V. Production of extruded snacks by utilization of watermelon (Citrullus vulgaris) seed flour. J. Postharvest Technol. 2019, 2019, 56-67. 